FIAN/TD-10/06

ITEP-TH-57/06

IHES-P $/ 06 / 43$

\title{
EXTENDED SEIBERG-WITTEN THEORY AND INTEGRABLE HIERARCHY
}

\author{
Andrei Marshakov ${ }^{1}$, Nikita A. Nekrasov ${ }^{\star 2}$ \\ 1 Lebedev Physics Institute and ITEP, Moscow, 117218 Russia \\ 2 Institut des Hautes Etudes Scientifiques, 91440 Bures-sur-Yvette, France ${ }^{\star \star}$ \\ 2 Department of Physics, Rutgers University, Piscataway, NJ 08544 USA
}

\begin{abstract}
The prepotential of the effective $\mathcal{N}=2$ super-Yang-Mills theory, perturbed in the ultraviolet by the descendents $\int d^{4} \theta \operatorname{tr} \boldsymbol{\Phi}^{k+1}$ of the single-trace chiral operators, is shown to be a particular tau-function of the quasiclassical Toda hierarchy. In the case of noncommutative $U(1)$ theory (or $U(N)$ theory with $2 N-2$ fundamental hypermultiplets at the appropriate locus of the moduli space of vacua) or a theory on a single fractional $D 3$ brane at the $A D E$ singularity the hierarchy is the dispersionless Toda chain, and we present its explicit solution. Our results generalise the limit shape analysis of Logan-Schepp and Vershik-Kerov, support the prior work [1], which established the equivalence of these $\mathcal{N}=2$ theories with the topological $\mathbf{A}$ string on $\mathbf{C P}^{1}$, and clarify the origin of the Eguchi-Yang matrix integral. In the higher rank case we find an appropriate variant of the quasiclassical tau-function, show how the Seiberg-Witten curve is deformed by Toda flows, and fix the contact term ambiguity.
\end{abstract}

\footnotetext{
* On leave of absence from: ITEP, Moscow, Russia

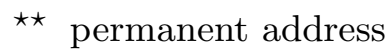




\section{TABLE OF CONTENTS}

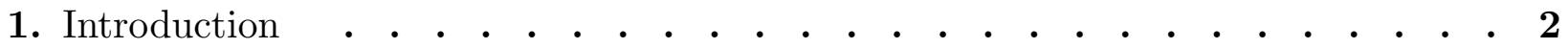

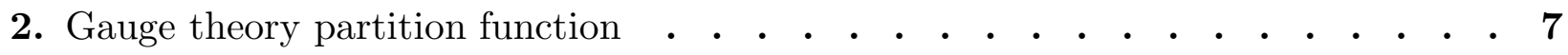

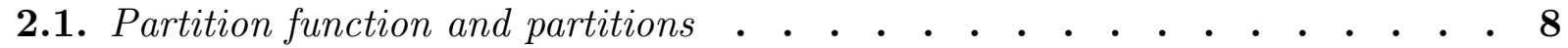

2.2. Fermions and Baker-Akhiezer functions . . . . . . . . . . . . . 10

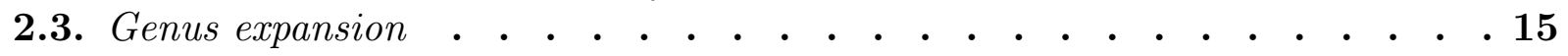

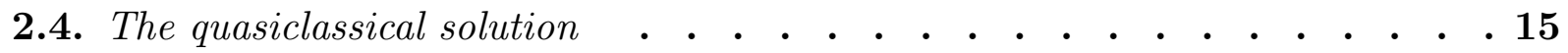

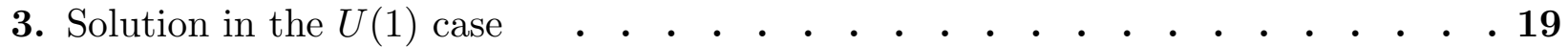

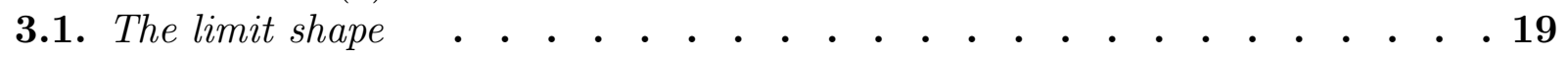

3.2. Toda as presentiment . . . . . . . . . . . . . . . . . . . . . 21

3.3. Symplectomorphisms and dispersionless hierarchy • • • • • • • • • 25

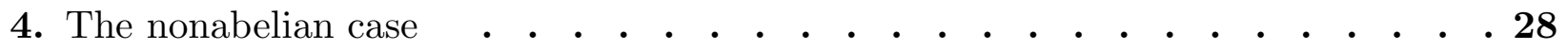

4.1. The energy and surface tension . • • • • • • • • • • • • • • • . 28

4.2. The curve . . . . . . . . . . . . . . . . . . . . . . . . . 28

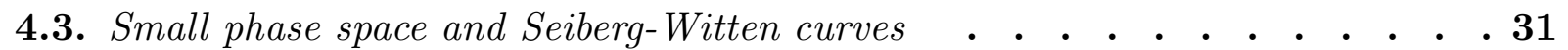

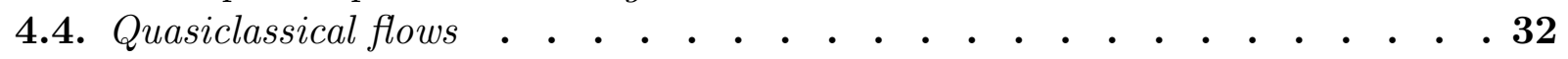

4.5. Instanton expansion with higher times . • . • • • • • • . • • . • . 34

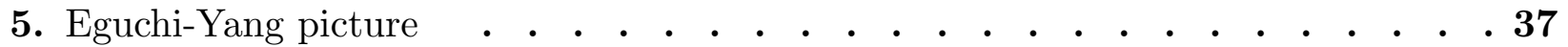

6. Conclusions and future directions . . . . . . . . . . . . . . . . . 40

Appendix A. Partitions and Chern characters . . . . . . . . . . . . 42 


\section{Introduction}

The supersymmetric $\mathcal{N}=2$ theories in two and four dimensions are quite interesting both for physicists and mathematicians. Their low-energy dynamics is rich, yet the supersymmetry is large enough to impose rather stringent constraints on the effective action.

Prepotential of effective theory

In quantum field theory one is interested in the low-energy effective action. The extended supersymmetry implies that all the terms with at most two derivatives and at most four fermions in the effective action are related, and can be expressed through derivatives of a single holomorphic quantity, a locally defined function $\mathcal{F}(\vec{a})$ on the moduli space $\mathcal{M}$ of vacua, the so-called prepotential.

In the recent years the exact calculations of the prepotential were performed, which utilized the fact that only the one-loop perturbative corrections about (an arbitrary) instanton solution summed over the instanton sectors, contribute to the prepotential. Actually, the prepotential itself is not so easy to calculate. However, it turns out that a certain "quantum corrected" prepotential

$$
Z(\vec{a}, \hbar)=\exp \sum_{g=0}^{\infty} F_{g}(\vec{a}) \hbar^{2 g-2}
$$

has a simple expression in terms of the gauge theory instantons. The leading term $\mathcal{F}(\vec{a})=$ $F_{0}(\vec{a})$ is the prepotential of the low-energy effective theory. The higher order terms, $F_{g}(\vec{a})$, describe the coupling of the theory to the $\mathcal{N}=2$ supergravity multiplet. In the string theory realisations of the $\mathcal{N}=2$ theory the terms $F_{g}(\vec{a})$ are computed by the genus $g$ string amplitudes.

The prepotential $F_{0}$ and the higher genus corrections $F_{g}$ are special in the sense that they determine the terms in the Lagrangian of effective theory, given by the integrals over half of the superspace, the so-called generalised F-terms. Formally one can consider the similar F-terms in the ultraviolet theory. The original microscopic Lagrangian

$$
L_{0}=\frac{1}{2} \int d^{4} \theta \tau_{0} \operatorname{tr} \boldsymbol{\Phi}^{2}+\frac{1}{2} \int d^{4} \bar{\theta} \bar{\tau}_{0} \operatorname{tr} \overline{\boldsymbol{\Phi}}^{2}
$$

can be deformed by adding the operators

$$
L_{\mathbf{t}}=L_{0}+\sum_{k>0} \frac{t_{k}}{k+1} \int d^{4} \theta \operatorname{tr} \Phi^{k+1}=L_{0}+\int d^{4} \theta \mathbf{t}(\boldsymbol{\Phi})
$$


where

$$
\Phi=\Phi+\theta \psi+\theta \theta F^{-}+\ldots
$$

is the vector superfield, and it is often convenient to work with the generating function

$$
\mathbf{t}(x)=\sum_{k>0} t_{k} \frac{x^{k+1}}{k+1}
$$

The bare couplings $\tau_{0}, \bar{\tau}_{0}$ are adjusted so as to produce the finite couplings in the effective Lagrangian, obtained by integrating out the high frequency modes.

\section{Renormalisation group flow}

We can reformulate our problem above as the calculation of the effect of the Wilsonian renormalisation group flow on the prepotential of the theory. One starts with the theory with $\mathcal{N}=2$ supersymmetry, which is determined by the ultraviolet prepotential $\mathcal{F}_{\mathrm{UV}}$, perturbed by arbitrary powers of the holomorphic operators

$$
\mathcal{F}_{\mathrm{UV}}=\frac{1}{2}\left(\tau_{0}+t_{1}\right) \operatorname{tr} \Phi^{2}+\sum_{k>0} t_{k} \frac{\operatorname{tr} \Phi^{k+1}}{k+1}
$$

and quadratic $\overline{\mathcal{F}}_{\mathrm{UV}}$, i.e.

$$
\overline{\mathcal{F}}_{\mathrm{UV}}=\frac{1}{2} \bar{\tau}_{0} \operatorname{tr} \bar{\Phi}^{2}
$$

Then one integrates out the fast modes, i.e. the perturbative fluctuations with momenta above certain scale $\mu$ as well as the non-perturbative modes, e.g. instantons (and fluctuations around them) of all sizes smaller then $\mu^{-1}$. The resulting effective theory has a derivative expansion in the powers $\frac{\partial^{2}}{\mu^{2}}$. The leading terms in the expansion are all determined, thanks to the $\mathcal{N}=2$ supersymmetry, by the effective prepotential $\mathcal{F}(\mu)$. As $\mu$ is lowered all the way down to zero, we arrive at the infrared prepotenial $\mathcal{F}_{\text {IR }}$ :

$$
\mathcal{F}_{\mathrm{UV}} \longrightarrow \mathcal{F}_{\mathrm{IR}}
$$

The supersymmetry considerations suggest that the renormalisation flows of $\mathcal{F}$ and $\overline{\mathcal{F}}$ proceed more or less independently from each other. Thus one can simplify the problem by taking the limit, $\bar{\tau}_{0} \rightarrow i \infty$, while $\mathcal{F}_{\mathrm{UV}}$ kept fixed. In this limit the path integral is dominated by the gauge instantons. The setup of [2] allows to evaluate their contribution, as well as the contribution of the fluctuations around the instantons, exactly. The price one pays is the introduction of extra parameters into the problem, some sort of the infrared 
cutoff, which we denote by $\hbar^{-2}$, since it appears to be a parameter of the loop expansion in dual topological string theory [1]. As we send $\hbar \rightarrow 0$, the infrared cutoff is removed, and the prepotential is recovered as the extensive part of the free energy, cf. (1.1). For details the reader is invited to consult [2][1][3].

\section{Prepotential and symplectic geometry}

The goal of this paper is to extract the generalised prepotential, $\mathcal{F}_{\mathrm{IR}}=\mathcal{F}(\vec{a}, \mathbf{t})$ as a function of the moduli of the vacua $\vec{a}$, and the higher Casimir couplings $\mathbf{t}$. It has been proposed in [4] (see also [5] and references therein) that the answer is given by tau-function of a quasiclassical or universal Whitham integrable hierarchy [6] and below we derive this hierarchy directly from the results of instanton calculus.

For fixed $\mathbf{t}$ the prepotential defines a generating function of a Lagrangian submanifold $\mathcal{L}_{\mathbf{t}}$ in the complex symplectic vector space $\mathbf{C}^{N}$, invariant under the action of a certain discrete subgroup $\Gamma$ of $\operatorname{Sp}(2 N, \mathbf{Z})$, the group of electric-magnetic dualities [7]. The deformations of such submanifolds are constrained by the considerations of duality, however at each order of deformation beyond the first one there are ambiguities:

$$
\begin{aligned}
\mathcal{F}(\vec{a}, \mathbf{t})= & \mathcal{F}(\vec{a}, 0)+\sum_{k>0} t_{k} u_{k+1} \\
& +\sum_{k, l>0} t_{k} t_{l}\left(-\frac{\partial u_{k+1}}{\partial a_{n}} \frac{\partial u_{l+1}}{\partial a_{m}} \frac{\partial}{\partial \tau_{m n}} \log \vartheta_{E}(0 \mid \tau)+\mathcal{C}_{k l}(u)\right)+\ldots
\end{aligned}
$$

where $u_{k} \sim\left\langle\operatorname{tr} \Phi^{k}\right\rangle$ are $\Gamma$-invariant functions on $\mathcal{L}_{0}$, i.e. the polynomials of the coefficients of Seiberg-Witten curve, $\mathcal{C}_{k l}(u)$ are some polynomials of $u$, called Losev-Shatashvili polynomials, and the precise definition of the theta constant and the theta characteristics in (1.7) is at the moment immaterial. While the choice of $u_{k}$ is more or less a matter of convention, the choice of Losev-Shatashvili polynomials $\mathcal{C}_{k l}(u)$ is physically important. The appearance of theta functions in (1.7) is dictated by the $\Gamma$-invariance and the extra terms $\mathcal{C}_{k l}(u)$ are constrained by the degree considerations [7], which predict that for small $k, l$, they vanish. However, to determine them one needs a microscopic theory, and below we show how a particular choice of microscopic theory determines all contact terms $\mathcal{C}_{k l}(u)$ in terms of quasiclassical integrable hierarchy. In particular, we shall see that Losev-Shatashvili polynomials $\mathcal{C}_{k l}(u)$ indeed vanish for $k, l<N$, i.e. exactly for the Casimirs which deform the Seiberg-Witten geometry for the $U(N)$ gauge theory. This distinguishes the quasiclassical hierarchy derived in this paper as directly coming from the microscopic instanton 
theory, compared to the previous attempts, proposed in [8], based on the analogies with Landau-Ginzburg models.

\section{Ensembles of partitions}

In particular, for the so-called noncommutative $U(1)$ theory (which supports instantons, $[9]$ ), or the theory on a single D3 brane in the background, which preserves only sixteen supercharges (so that the theory on the brane has only eight supercharges), the instanton partition function $Z(a, \hbar, \mathbf{t}), \mathbf{t}=\left(t_{1}, t_{2}, \ldots\right)$, can be shown to be given by the sum over the Young diagrams, i.e. over the partitions [2][1][3]:

$$
Z(a, \mathbf{t}, \hbar)=\sum_{\lambda} \frac{\mathbf{m}_{\lambda}^{2}}{\left(-\hbar^{2}\right)^{|\lambda|}} \exp \frac{1}{\hbar^{2}} \sum_{k>0} t_{k} \frac{\operatorname{ch}_{k+1}(a, \lambda)}{k+1}
$$

(we shall remind the relevant notions of the theory of partitions in the Appendix). This theory can also be realised at a special point on the moduli space of $U(N)$ gauge theory with $2 N-2$ fundamental hypermultiplets ${ }^{\dagger}$. If the theory has the gauge group $U(N)$, e.g. it is realised on the stack of $N$ fractional D3 branes, the corresponding partition function is given by the generalisation of (1.8):

$$
Z(\vec{a}, \mathbf{t}, \hbar)=Z^{\text {pert }}(\vec{a}, \mathbf{t}, \hbar) \sum_{\vec{\lambda}}(\mathbf{m}(\vec{a}, \vec{\lambda}, \hbar))^{2}(-1)^{|\vec{\lambda}|} \exp \frac{1}{\hbar^{2}} \sum_{k>0} t_{k} \frac{\operatorname{ch}_{k+1}(\vec{a}, \vec{\lambda})}{k+1}
$$

where $\mathbf{m}(\vec{a}, \vec{\lambda}, \hbar)$ is the $U(N)$ generalisation of Plancherel measure [2][3] and $Z^{\text {pert }}(\vec{a}, \mathbf{t}, \hbar)$ is the perturbative partition function.

\section{Limit shape and Toda chain}

We shall evaluate the sums like (1.8) by the analogue of the saddle point method. The limit $\hbar \rightarrow 0$ of the sum (1.8) is dominated by a partition $\lambda_{*}$, of a large size $\sim \hbar^{-2}$. The shape of the Young diagram of this partition, the so-called limit shape, is found by extremizing the effective energy functional. When all but the first coupling $t_{1}$ are set to zero, this limit shape is the celebrated "arcsin law" curve, found by Vershik-Kerov and Logan-Shepp

$\dagger$ In a certain sense, all the instanton contributions in the "U(1)" theory are the artefacts of the imbedding of the theory into the theory with rich ultraviolet structure. In the terminology of [10] these are "freckled instantons" and their contribution must be subtracted by the appropriate "mirror map". See also [11] for the related and more detailed discussion, and [12] for the related $\mathcal{N}=1$ considerations. 
[13][14][15][16]. Our main claim for the rank $N=1$ is that the evolution of the limit shape under the higher Casimirs is governed by the quasiclassical Toda hierarchy, and we present the corresponding solution explicitly. This result, which we prove in the section $\underline{3}$, gives a further confirmation of the claim of the previous work [1] of A. S. Losev and the authors, that the BPS sector of the rank one $\mathcal{N}=2$ gauge theory is equivalent to the stationary sector of the $\mathbf{C} \mathbf{P}^{1}$ Gromov-Witten theory. The dispersionless Toda hierarchy is well-known to describe the genus zero part of that theory (see, e.g. [17]).

In the section $\underline{4}$ we describe the non-abelian theory. We find that the corresponding limit shape is described by the Krichever quasiclassical tau-function [6] associated with a family of hyperelliptic curves with two marked points

$$
y^{2}=\prod_{l=1}^{N}\left(z-x_{l}^{+}(\vec{a}, \mathbf{t})\right)\left(z-x_{l}^{-}(\vec{a}, \mathbf{t})\right)
$$

Interestingly enough, for nonvanishing $\mathbf{t}$ this family extends outside the family of SeibergWitten curves. The answer is encoded in (1.10) and a particular $(1,0)$-differential $d \Sigma$,

$$
d \Sigma=\frac{\sigma(z ; \vec{a}, \mathbf{t}) d z}{y},
$$

holomorphic outside the points $P_{ \pm}$where $z=\infty$, which obeys certain normalisation conditions. We demonstrate how the instanton corrections (for nonvanishing $\mathbf{t}$ ) can be extracted from the generalised Seiberg-Witten geometry, and derive equation (1.7) from the "first principles". In the section $\underline{5}$ discuss the relation of our approach with the Eguchi-Yang matrix integral and propose a possible way to extend this relation beyond the quasiclassical theory. 


\section{Gauge theory partition function}

We study $\mathcal{N}=2$ gauge theory in the self-dual $\Omega$-background. The setup and the relevant physics are reviewed in [2][1][3] so we just briefly discuss it here. The gauge theory path integral is saturated by instantons, and $\mathcal{N}=2$ supersymmetry cancels the contribution of fluctuations, so that effectively one has to integrate "unities" over the corresponding moduli spaces of the instanton solutions. These moduli spaces can be described by the ADHM construction, and the corresponding integrals can be computed via equivariant localisation technique in nontrivial $\Omega$-background [2]. As a result, the gauge theory partition function can be presented effectively in one of the following ways:

1) The sum over instantons can be interpreted as a Van-der-Vaals gas in one dimensions: the integrals over the instanton moduli space of charge $k$ can be reduced, via ADHM construction and equivariant localisation a la [18] to the grand canonical ensemble of a one-dimensional gas of particles

$$
\begin{aligned}
& Z\left(\vec{a}, \mathbf{t}, \epsilon_{1,2}\right)=Z^{\text {pert }}\left(\vec{a}, \mathbf{t}, \epsilon_{1,2}\right) \times \\
& \times \sum_{k=0}^{\infty} \frac{1}{k !}\left(\frac{\epsilon_{1}+\epsilon_{2}}{\epsilon_{1} \epsilon_{2}}\right)^{k} \oint \prod_{I=1}^{k} d \phi_{I} \exp \left(-\frac{1}{\epsilon_{1} \epsilon_{2}} U\left(\phi_{I}\right)\right) \times \\
& \times \prod_{I<J} \exp \left(-\frac{1}{\epsilon_{1} \epsilon_{2}} V\left(\phi_{I}-\phi_{J}\right)\right) \\
& \text { where } U(x)=\mathbf{t}(x)+\mathbf{t}\left(x+\epsilon_{1}+\epsilon_{2}\right)-\mathbf{t}\left(x+\epsilon_{1}\right)-\mathbf{t}\left(x+\epsilon_{2}\right)+ \\
& +\epsilon_{1} \epsilon_{2} \sum_{l=1}^{N} \log \left(\left(x-a_{l}\right)^{2}-\frac{1}{4}\left(\epsilon_{1}+\epsilon_{2}\right)^{2}\right) \\
& V(x)=-\epsilon_{1} \epsilon_{2} \log \frac{x^{2}\left(x^{2}-\left(\epsilon_{1}+\epsilon_{2}\right)^{2}\right)}{\left(x^{2}-\epsilon_{1}^{2}\right)\left(x^{2}-\epsilon_{2}^{2}\right)}
\end{aligned}
$$

interacting via pair-wise Van-der-Vaals kind of potential, in the presence of $N$ (for $U(N)$ theory) sources. Here $\epsilon_{1,2}$ are parameters of the $\Omega$-background, which can be, after calculating integrals in (2.1) via residues, put to be $\epsilon_{1}=-\epsilon_{2}=\hbar$.

2) The gauge theory partition function can be shown to be equal to the partition function of the statistical model of random partitions. For $U(N)$ gauge theory one deals with the ensemble of $N$-tuples of partitions $\vec{\lambda}=\left(\lambda_{1}, \ldots, \lambda_{N}\right)$, and the partition function of the gauge theory is $[2][3]$ :

$$
Z(\vec{a}, \mathbf{t}, \hbar)=Z^{\text {pert }}(\vec{a}, \mathbf{t}, \hbar) \cdot \sum_{\vec{\lambda}} \mathbf{m}^{2}(\vec{a}, \vec{\lambda}, \hbar)(-1)^{|\vec{\lambda}|} \exp \frac{1}{\hbar^{2}} \sum_{k=1}^{\infty} t_{k} \frac{\operatorname{ch}_{k+1}(\vec{a}, \vec{\lambda})}{k+1}
$$


3) The gauge theory partition function can be also written as a path integral in the theory of a free chiral fermion on a Riemann sphere [1], or, via bosonisation, as a path integral in the theory of a free boson $\varphi$ with the action:

$$
\mathcal{S}=\frac{1}{4 \pi} \int_{\mathbf{S}^{2}} \partial \varphi \bar{\partial} \varphi+\frac{1}{\hbar} \oint_{C_{\varepsilon}} J w^{-1}+\frac{1}{\hbar} \oint_{C_{1 / \varepsilon}} J w+\oint_{C_{1}} \sum_{k} t_{k} \hbar^{k-1}\left(J^{k+1}+\ldots\right)
$$

with the boson $\varphi$ normalised so that it takes values in a circle of finite circumference. In (2.3) $C_{r}$ denotes a circle $|w|=r$, and $\varepsilon \downarrow 0$. Similar path integrals were studied before in the context of so called conformal matrix models [19], though with very different properties of genus expansion [20]. In this paper we shall concentrate on the quasiclassical computations in the theory (2.3).

We start with reminding the basic facts about partitions and free fermions.

\subsection{Partition function and partitions}

The partition $\lambda$ of the size $|\lambda|=\lambda_{1}+\lambda_{2}+\ldots+\lambda_{\ell_{\lambda}}$ is a non-increasing sequence of non-negative integers $\lambda_{i} \in \mathbf{Z}_{\geq 0}$ (some details and examples are collected in Appendix A):

$$
\lambda=\left(\lambda_{1} \geq \lambda_{2} \geq \lambda_{3} \ldots \geq \lambda_{\ell_{\lambda}}>\lambda_{\ell_{\lambda}+1}=\lambda_{\ell_{\lambda}+2}=\ldots=0\right)
$$

For our purposes it is convenient to encode the partition $\lambda$ in the so-called profile function $f_{\lambda}(x)$, which is a piece-wise linear function, given by:

$$
\begin{aligned}
f_{\lambda}(x)=|x-a|+ & \\
+\sum_{i=1}^{\infty} \quad\left|x-a-\hbar\left(\lambda_{i}-i+1\right)\right|-\left|x-a-\hbar\left(\lambda_{i}-i\right)\right| & -|x-a-\hbar(1-i)|+|x-a+\hbar i|
\end{aligned}
$$

The Chern character of the "universal sheaf" $\operatorname{ch}(\mathcal{E})$ (at the fixed point in instanton moduli space, characterized by partition $\lambda$, see [1][2] and references therein) is essentially the Fourier transform of the profile function:

$$
\operatorname{ch}(\mathcal{E})=\frac{1}{2} \int d x f_{\lambda}^{\prime \prime}(x) e^{u x}=e^{u a}\left(1+\left(1-e^{-u \hbar}\right) \sum_{i=1}^{\infty} e^{u \hbar(1-i)}\left(e^{u \hbar \lambda_{i}}-1\right)\right)
$$

hence for the coefficients of its expansion

$$
\operatorname{ch}(\mathcal{E})=\left(e^{\frac{\hbar u}{2}}-e^{-\frac{\hbar u}{2}}\right) \operatorname{ch}_{\lambda}\left(\frac{a}{\hbar}, \hbar u\right)=\sum_{k=0}^{\infty} \frac{u^{k}}{k !} \operatorname{ch}_{k}(a, \lambda)
$$


which enter the formula for the statistical weight of the partition $\lambda$ in the ensemble (1.8), one has

$$
\operatorname{ch}_{k}(a, \lambda)=\frac{1}{2} \int d x f_{\lambda}^{\prime \prime}(x) x^{k} \sim \sum_{i=1}^{\infty}\left(\left(a+\hbar\left(\lambda_{i}-i+1\right)\right)^{k}-\left(a+\hbar\left(\lambda_{i}-i\right)\right)^{k}\right)
$$

In the nonabelian case, for the gauge group $U(N)$, the universal sheaf $\mathcal{E}$ splits as a sum of $N$ rank one sheaves $\mathcal{E}_{l}$. Accordingly, at the fixed point of the torus action on the moduli space of instantons, corresponding to the $N$-tuple of partitions $\vec{\lambda}$ :

$$
\operatorname{ch}(\mathcal{E})=\left(e^{\frac{\hbar u}{2}}-e^{-\frac{\hbar u}{2}}\right) \sum_{l=1}^{N} e^{u a_{l}} \operatorname{ch}_{\lambda^{(l)}}(0, \hbar u)=\sum_{k=0}^{\infty} \frac{u^{k}}{k !} \operatorname{ch}_{k}(\vec{a}, \vec{\lambda})
$$

with

$$
\operatorname{ch}_{k}(\vec{a}, \vec{\lambda})=\sum_{l=1}^{N} \operatorname{ch}_{k}\left(a_{l}, \lambda_{l}\right)
$$

For the empty partitions $\lambda^{(l)}=\emptyset$ the Chern character (2.9) reduces to the generating function of the vacuum expectation values of the single trace operators $\operatorname{tr} \Phi^{k}$, in the absence of quantum corrections.

Plancherel measure and the profile of the partition

The Plancherel measure

$$
\mathbf{m}_{\lambda}=\prod_{i<j} \frac{\lambda_{i}-\lambda_{j}+j-i}{j-i}=\prod_{i=1}^{\ell_{\lambda}} \frac{\left(\ell_{\lambda}-i\right) !}{\left(\ell_{\lambda}+\lambda_{i}-i\right) !} \prod_{1 \leq i<j \leq \ell_{\lambda}} \frac{\lambda_{i}-\lambda_{j}+j-i}{j-i}
$$

can also be expressed in terms of the profile function $f_{\lambda}(x)$ :

$$
\frac{\mathbf{m}_{\lambda}^{2}}{\left(-\hbar^{2}\right)^{|\lambda|}}=\exp \left(-\frac{1}{4} \int_{x_{1}>x_{2}} d x_{1} d x_{2} f_{\lambda}^{\prime \prime}\left(x_{1}\right) f_{\lambda}^{\prime \prime}\left(x_{2}\right) \gamma_{\hbar}\left(x_{1}-x_{2}\right)\right)
$$

where the kernel $\gamma_{\hbar}(x)$ solves the following difference equation:

$$
\gamma_{\hbar}(x+\hbar)+\gamma_{\hbar}(x-\hbar)-2 \gamma_{\hbar}(x)=\log x^{2}
$$

and is given by the asymptotic series:

$$
\gamma_{\hbar}(x)=\frac{1}{\hbar^{2}}\left(\mathbf{F}(x)-\frac{\hbar^{2}}{12} \mathbf{F}^{(2)}(x)+\frac{\hbar^{4}}{240} \mathbf{F}^{(4)}(x)-\frac{\hbar^{6}}{6048} \mathbf{F}^{(6)}(x)+\ldots\right)
$$


determined by its leading term, or the function:

$$
\mathbf{F}(x)=x^{2}\left(\log x-\frac{3}{2}\right)
$$

which enters the perturbative prepotential of pure $\mathcal{N}=2$ super-Yang-Mills theory. The finite difference derivative of $\gamma_{\hbar}(x)$ is related to the $\Gamma$-function:

$$
\gamma_{\hbar}\left(x+\frac{\hbar}{2}\right)-\gamma_{\hbar}\left(x-\frac{\hbar}{2}\right)=\log \left(\hbar^{\frac{x}{\hbar}} \Gamma\left(\frac{x}{\hbar}+\frac{1}{2}\right)\right)
$$

In the $\hbar \rightarrow 0$ limit (2.13) just turns into $\mathbf{F}^{\prime \prime}(x)=2 \log x$. The function $\gamma_{\hbar}(x)$ is related to the generalized Riemann zeta function:

$$
\gamma_{\hbar}(x)=-\zeta^{\prime}(-1)+\frac{1}{12} \log \hbar+\left.\frac{d}{d s}\right|_{s=0} \frac{1}{\Gamma(s)} \int_{0}^{\infty} \frac{d t}{t} t^{s} \frac{e^{-t x}}{\left(e^{t \hbar}-1\right)\left(e^{-t \hbar}-1\right)}
$$

The $\zeta^{\prime}(-1)$ and $\log \hbar$ terms in $(2.17)$ are chosen so as to ensure $\gamma_{\hbar}(0)=0$. These corrections do not give any contribution to quasiclassical part and can be therefore simply absorbed into normalisation (in particular we did not write them in (2.14)).

\subsection{Fermions and Baker-Akhiezer functions}

The gauge theory partition function (1.8),(1.9) can be compactly written as a matrix element in the infinite wedge representation of the group $G L(\infty)$, in other words, it has a free fermion representation [1][3](see also [21]). We shall recall it now in order to motivate

the introduction of some quasiclassical objects, like the multi-valued functions $S(z)$ and $\Phi(z)$, which will be the main tool for solving our problem. As we shall see, they appear in the asymptotic expansion of the fermionic one-point functions, or the Baker-Akhiezer functions (cf. [22][23]).

Free fermions and partitions

Introduce the free fermion fields, $\psi(w), \widetilde{\psi}(w)$ :

$$
\begin{aligned}
& \psi(w)=\sum_{r \in \mathbf{Z}+\frac{1}{2}} \psi_{r} w^{-r}\left(\frac{d w}{w}\right)^{\frac{1}{2}} \\
& \widetilde{\psi}(w)=\sum_{r \in \mathbf{Z}+\frac{1}{2}} \widetilde{\psi}_{r} w^{r}\left(\frac{d w}{w}\right)^{\frac{1}{2}}
\end{aligned}
$$


where $\left\{\psi_{r}, \widetilde{\psi}_{s}\right\}=\delta_{r s}$. The vacuum with the charge $M$ is defined as:

$$
|M\rangle=|M ; \emptyset\rangle=\psi_{-M+\frac{1}{2}} \psi_{-M+\frac{3}{2}} \psi_{-M+\frac{5}{2}} \cdots
$$

and is annihilated by the fermion harmonics:

$$
\begin{aligned}
& \psi_{r}|M\rangle=0, \quad r>-M, \\
& \widetilde{\psi}_{r}|M\rangle=0, \quad r<-M
\end{aligned}
$$

We always use the normal ordering : (..) : with respect to the vacuum $|0\rangle$ :

$$
\begin{array}{lll}
: \psi_{r} \widetilde{\psi}_{s}:=\psi_{r} \widetilde{\psi}_{s}, & s<0 \\
: \psi_{r} \widetilde{\psi}_{s}:=-\widetilde{\psi}_{s} \psi_{r}, & r>0
\end{array}
$$

Let us introduce the $W_{1+\infty}$ algebra operators:

$$
\begin{aligned}
\mathbf{W}_{k+1}= & -\frac{\hbar^{k}}{k+1} \oint: \widetilde{\psi}\left(\left(D+\frac{1}{2}\right)^{k+1}-\left(D-\frac{1}{2}\right)^{k+1}\right) \psi: \\
& =\frac{\hbar^{k}}{k+1} \sum_{r \in \mathbf{Z}+\frac{1}{2}}\left[\left(-r+\frac{1}{2}\right)^{k+1}-\left(-r-\frac{1}{2}\right)^{k+1}\right]: \psi_{r} \widetilde{\psi}_{r}:
\end{aligned}
$$

where $D=w \partial_{w}$ and $k \geq 0$. For example,

$$
\mathbf{W}_{1}=-J_{0}, \mathbf{W}_{2}=\hbar L_{0}, \quad \ldots
$$

i.e. $J_{0}$ is the zero mode of the $U(1)$ current,

$$
\begin{aligned}
J(w) & =: \widetilde{\psi}(w) \psi(w):=\sum_{k \in \mathbf{Z}} J_{k} w^{-k} \frac{d w}{w} \\
J_{k} & =\sum_{r \in \mathbf{Z}+\frac{1}{2}}: \widetilde{\psi}_{r} \psi_{r+k}:
\end{aligned}
$$

while the $L_{0}$ Virasoro generator is the zero mode of the stress-energy tensor:

$$
\begin{aligned}
T(w) & =-: \widetilde{\psi}(w) d \psi(w):=\sum_{k \in \mathbf{Z}} L_{k} w^{-k}\left(\frac{d w}{w}\right)^{2} \\
L_{k} & =\sum_{r \in \mathbf{Z}+\frac{1}{2}} r: \widetilde{\psi}_{r} \psi_{r+k}:
\end{aligned}
$$


The importance of the fermions is the relation between the partitions and the excited states in the fermion Fock space:

$$
|M ; \lambda\rangle=\psi_{-M+\frac{1}{2}-\lambda_{1}} \psi_{-M+\frac{3}{2}-\lambda_{2}} \psi_{-M+\frac{5}{2}-\lambda_{3}} \ldots \psi_{-M-\frac{1}{2}+i-\lambda_{i}} \cdots
$$

The operators $\mathbf{W}_{k}$ introduced in (2.22) are diagonal in the basis of "partition" states:

$$
\mathbf{W}_{k}|M ; \lambda\rangle=\frac{1}{\hbar k} \operatorname{ch}_{k}(\hbar M, \lambda)|M ; \lambda\rangle
$$

with the eigenvalues given by (2.8). In what follows, we shall also use the formula [24][25]:

$$
e^{\frac{J_{-1}}{\hbar}}|M ; \emptyset\rangle=\sum_{\lambda} \mathbf{m}_{\lambda} \hbar^{-|\lambda|}|M ; \lambda\rangle
$$

and its direct consequence

$$
\widetilde{\psi}_{-r} e^{\frac{J_{-1}}{\hbar}}|M+1 ; \emptyset\rangle=\hbar^{-r-\frac{1}{2}} \sum_{\lambda} \frac{\mathbf{m}_{\lambda}}{\hbar^{|\lambda|}} \prod_{i=1}^{\infty} \frac{i-\lambda_{i}+r-\frac{1}{2}-M}{i}|M ; \lambda\rangle
$$

where the sum is now taken over all partitions do not containing an eigenvalue, corrseponding to $r$-th fermionic mode, which is automatically taken into account vanishing of the product factor. The infinite product in (2.29) is actually finite

$$
\prod_{i=1}^{\infty} \frac{i-\lambda_{i}+r-\frac{1}{2}-M}{i}=\frac{1}{\Gamma\left(r+\frac{1}{2}-M\right)} \prod_{i=1}^{\ell_{\lambda}} \frac{i-\lambda_{i}+r-\frac{1}{2}-M}{i+r-\frac{1}{2}-M}
$$

\section{Baker-Akhiezer functions}

The sum over partitions (1.8) can be compactly written as a matrix element in the theory of free fermions [1][3]:

$$
Z(a, \mathbf{t}, \hbar)=\left\langle M\left|e^{-\frac{J_{1}}{\hbar}} e^{\frac{1}{\hbar} \sum_{k>0} t_{k} \mathbf{W}_{k+1}} e^{\frac{J_{-1}}{\hbar}}\right| M\right\rangle=\sum_{\lambda} \frac{\mathbf{m}_{\lambda}^{2}}{\left(-\hbar^{2}\right)^{|\lambda|}} e^{\frac{1}{\hbar^{2}} \sum_{k>0} t_{k} \frac{\mathrm{ch}_{k+1}(a, \lambda)}{k+1}}
$$

where $a=\hbar M$ for $M \in \mathbf{Z}$, and the second equality follows from (2.28), (2.27). Let $r \in \mathbf{Z}+\frac{1}{2}$ and set $z=\hbar r$. Consider the Baker-Akhiezer function, or the following matrix element:

$$
\widetilde{\Psi}(z, a, \mathbf{t}, \hbar)=\frac{1}{Z(a, \mathbf{t}, \hbar)}\left\langle M\left|e^{-\frac{J_{1}}{\hbar}} \widetilde{\psi}_{-r} e^{\frac{1}{\hbar} \sum_{k>0} t_{k} \mathbf{W}_{k+1}} e^{\frac{J_{-1}}{\hbar}}\right| M+1\right\rangle
$$


Using (2.28), (2.29) it can be expanded into a sum over partitions

$$
\begin{aligned}
\widetilde{\Psi}(z, a, \mathbf{t}, \hbar)= & \frac{\hbar^{-r-\frac{1}{2}}}{Z(a, \mathbf{t}, \hbar)} \exp \frac{1}{\hbar^{2}} \sum_{k>0} \frac{t_{k} \hbar^{k}}{k+1}\left(\left(r+\frac{1}{2}\right)^{k+1}-\left(r-\frac{1}{2}\right)^{k+1}\right) \cdot \\
& \cdot \sum_{\lambda} \mathbf{m}_{\lambda}^{2}\left(-\hbar^{-2}\right)^{|\lambda|} e^{\frac{1}{\hbar^{2}} \sum_{k>0} t_{k} \frac{\mathrm{ch}_{k+1}(a, \lambda)}{k+1}} \prod_{i=1}^{\infty} \frac{i-\lambda_{i}+r-\frac{1}{2}-M}{i}
\end{aligned}
$$

The latter can be obtained from the partition function (2.31) by the shift of times $t_{k} \rightarrow$ $t_{k}-\delta_{k}(\hbar, r):$

$$
\begin{array}{r}
\widetilde{\Psi}(z, a, \mathbf{t}, \hbar)=\hbar^{-r-\frac{1}{2}} \exp \frac{1}{\hbar^{2}} \sum_{k>0} \frac{t_{k} \hbar^{k}}{k+1}\left(\left(r+\frac{1}{2}\right)^{k+1}-\left(r-\frac{1}{2}\right)^{k+1}\right) \\
\cdot \frac{e^{M \Gamma^{\prime}\left(r+\frac{1}{2}\right) / \Gamma\left(r+\frac{1}{2}\right)}}{\Gamma\left(r+\frac{1}{2}\right)} \frac{Z(a, \hbar, \mathbf{t}-\delta)}{Z(a, \hbar, \mathbf{t})}
\end{array}
$$

defined via the generation function

$$
\frac{1}{\hbar^{2}} \sum_{k>0} \delta_{k} \frac{x^{k+1}}{k+1}=\log \frac{\Gamma\left(r+\frac{1}{2}-\frac{x}{\hbar}\right)}{\Gamma\left(r+\frac{1}{2}\right)}+\frac{x}{\hbar} \log \frac{\Gamma^{\prime}\left(r+\frac{1}{2}\right)}{\Gamma\left(r+\frac{1}{2}\right)}
$$

From (2.34) one gets, that asymptotically at $\hbar \rightarrow 0$ and $\hbar r=z \rightarrow \infty$

$$
\widetilde{\Psi}(z, a, \mathbf{t}, \hbar) \sim \exp \frac{S(z, a, \mathbf{t})}{\hbar}
$$

with the singularity

$$
S(z, a, \mathbf{t})=\sum_{k>0} t_{k} z^{k}-z(\log z-1)+a \log z+\ldots
$$

while the nonsingular at $z \rightarrow \infty$ terms in (2.37) are expressed, as follows from (2.34), in terms of the first derivatives of $\log Z(a, \mathbf{t}, \hbar)$ w.r.t. t-variables. The essential singularity (2.37) contains the Eguchi-Yang term $z(\log z-1)$, (cf. [17]), coming from the Gamma function $\log \Gamma(z) \sim z(\log z-1)$ in (2.34). The fact that the regular term of the first order in $z, t_{1} z$ in $(2.37)$ is accompanied by the $z(\log z-1)$ term, of the degree $1+\epsilon$, in a sense, is the consequence of the asymptotic freedom of the noncommutative $U(1)$ theory (see [3] for the discussion of this phenomenon). In the $U(N)$ case we shall see the analogous shift, $t_{1} \rightarrow t_{1}-N \log z$, in agreement with the exact coefficient, $N$, of the beta function of $U(N)$ super-Yang-Mills theory [26]. 
One can also study the fermion matrix element in the coordinate representation:

$$
\begin{gathered}
\widehat{\Psi}(w, a, \mathbf{t}, \hbar)=\frac{1}{Z(a, \mathbf{t}, \hbar)}\left\langle M\left|e^{-\frac{J_{1}}{\hbar}} e^{\frac{1}{\hbar} \sum_{k>0} t_{k} \mathbf{W}_{k+1}} \widetilde{\psi}(w) e^{\frac{J_{1}}{\hbar}}\right| M+1\right\rangle= \\
=\sum_{r \in \mathbf{Z}+\frac{1}{2}} w^{-r} \widetilde{\Psi}(r \hbar, a, \mathbf{t}, \hbar)
\end{gathered}
$$

Asymptotically, (2.38) gives

$$
\widehat{\Psi}(w, a, \mathbf{t}, \hbar) \sim \exp \frac{\Sigma(w, a, \mathbf{t})}{\hbar}
$$

with the quasiclassical phase:

$$
\Sigma(w, a, \mathbf{t})=S(z, a, \mathbf{t})-z \log w, \quad \frac{d S(z, a, \mathbf{t})}{d z}=\log w
$$

related to (2.36) by the Legendre transform, while (2.38) can be thought as an integral duality transformation [27] for the Baker-Akhiezer functions. Note (for fixed $(a, \mathbf{t})$ ):

$$
d \Sigma=-z \frac{d w}{w}
$$

The nonabelian theory partition function also has a representation in terms of free fermions. For the gauge group $U(N)$ one uses $N$ flavours, $\psi^{(l)}(w), l=1, \ldots, N$. For the special values of the vacuum expectation value of the Higgs scalar $\Phi, a_{l}=\hbar\left(M_{l}+\rho_{l}\right)$, where $M_{l} \in \mathbf{Z}$ are integers, $\rho_{l}=\frac{1}{N}(N+1-2 l)$, the partition function of the nonabelian theory can be related to that of the abelian one by means of the procedure of blending of partitions. In the language of free fermions this is the following procedure:

$$
\Psi\left(w^{\frac{1}{N}}\right)=\frac{1}{\sqrt{N}} \sum_{l=1}^{N} \psi^{(l)}(w) w^{-\rho_{l}}
$$

The single free fermion $\Psi$ is produced out of $N$ free fermions $\psi^{(l)}, l=1, \ldots, N$ living on the $w$-space. The fermion $\Psi$ lives on the $N$-fold cover of the original space, which in quasiclassical limit turns into a spectral curve.

The spectral curve for a nonabelian theory will be explicitly described below. On a small phase space (with only $t_{1}$ nonvanishing) it coincides with the Seiberg-Witten curve, but when higher Casimirs are switched on, it goes beyond the Seiberg-Witten family, still being a hyperelliptic curve for the $U(N)$ gauge theory. This curve corresponds to a quasiclassical hierarchy [6] of the Toda type, and we shall see that co-ordinate $z=\hbar r$, corresponding to the Baker-Akhiezer functions of the type (2.36) is distinguished from the point of view of this hierarchy. 


\subsection{Genus expansion}

The partition function (1.8), (1.9) has the same genus expansion, as in (1.1), but with the switched on times $\mathbf{t}$. There are various interpretations of this expansion, which will be presented in detail elsewhere [28].

a.) Gauge theory interpretation in four dimensions: the $\Omega$-background acts as a "smart box" with parameter $\hbar^{2}$ being the inverse volume, prepotential as the extensive part of the free energy, the higher terms $F_{g}$ being the finite size effects.

б.) Topological string interpretation: in the geometrical engineering setup $F_{g}$ 's are the genus $g$ topological string amplitudes on a local Calabi-Yau manifold.

в.) Van-der-Vaals gas in one dimensions: the $\hbar$-expansion becomes the Mayer diagram expansion [28] in this case.

г.) We can view (2.3) as the quantum field theory and perform the standard Feynman diagram expansion around the Gaussian action $\frac{1}{4 \pi} \int_{\mathbf{S}^{2}} \partial \varphi \bar{\partial} \varphi$ with the vertices of valency $p$ weighted with the weight $\hbar^{p-2}$, so that the sources $\oint \partial \varphi w^{ \pm 1}$ have the weight $\hbar^{-1}$, the "mass term" $t_{1} \oint(\partial \varphi)^{2}$ having no $\hbar$-dependent factor at all, etc. The weight of the Feynman diagram $\gamma$ with $v_{p}(\gamma)$ vertices of valency $p$ would be, then

$$
\hbar^{\sum_{p}(p-2) v_{p}(\gamma)}=\hbar^{2(E(\gamma)-V(\gamma))}=\hbar^{2 g-2}
$$

where

$$
E(\gamma)=\frac{1}{2} \sum_{p} p v_{p}(\gamma)
$$

is the number of edges, and

$$
V(\gamma)=\sum_{p} v_{p}(\gamma)
$$

is the number of vertices, and $g$ is the number of loops. In this way one can make contact with the string loop expansion.

\subsection{The quasiclassical solution}

The partition functions (1.8)(1.9) should be viewed as the partition functions of an instanton gas in the "box" of size $\hbar^{-2}$. We are interested in the free energy of the instanton gas, per unit volume:

$$
\mathcal{F}(\vec{a}, \mathbf{t})=\operatorname{Lim}_{\hbar \rightarrow 0} \hbar^{2} \log Z(\vec{a}, \hbar, \mathbf{t})
$$




\section{Energy and entropy}

The evaluation of the asymptotics (2.43) is greatly facilitated by the observation that in the limit where $\hbar \rightarrow 0$ with the rest of the parameters kept fixed, the sum over the partitions $\lambda$ (or $N$-tuples $\vec{\lambda}$ ) is dominated by the single partition of the very large size $\sim \hbar^{-2}$ [3]. To determine this partition and to evaluate (2.43) one can use the geometric representation of partitions, Young diagrams. In the limit $\hbar \rightarrow 0$ the boundary of Young diagram of the master partition becomes a continuous curve. This curve, e.g. the profile $f_{\lambda}(x)$, can be found by maximizing the weight of the partition $\lambda$ in the sum (1.8). The Plancherel measure (2.12) and the Chern characters (2.8) in (1.8) combine together into the following energy functional

$$
E(\lambda, \hbar)=\frac{1}{4} \int_{x_{1}>x_{2}} d x_{1} d x_{2} f_{\lambda}^{\prime \prime}\left(x_{1}\right) f_{\lambda}^{\prime \prime}\left(x_{2}\right) \gamma_{\hbar}\left(x_{1}-x_{2}\right)-\frac{1}{2 \hbar^{2}} \int d x f_{\lambda}^{\prime \prime}(x) \mathbf{t}(x)
$$

so that

$$
Z(a, \mathbf{t}, \hbar)=\sum_{\lambda} e^{-E(\lambda, \hbar)}
$$

Expanding the energy $E(\lambda, \hbar)$ in $\hbar$ (assuming $f_{\lambda}(x) \rightarrow f(x)$ ):

$$
E(\lambda, \hbar) \sim \frac{1}{\hbar^{2}} \mathcal{E}[f]
$$

where

$$
\mathcal{E}[f]=\frac{1}{4} \int_{x_{1}>x_{2}} d x_{1} d x_{2} f^{\prime \prime}\left(x_{1}\right) f^{\prime \prime}\left(x_{2}\right) \mathbf{F}\left(x_{1}-x_{2}\right)-\frac{1}{2} \int d x f^{\prime \prime}(x) \mathbf{t}(x),
$$

we can approximate the partition function as:

$$
Z(a, \mathbf{t}, \hbar) \sim \exp \left(-\frac{1}{\hbar^{2}} \mathcal{E}[f]\right)
$$

Indeed, the entropy contribution, i.e. the number of partitions $\lambda$, whose profiles $f_{\lambda}(x)$ differ from $f(x)$ by $O(\hbar)$, is of the order of

$$
\exp \left(\frac{1}{\hbar} L\right)
$$

where $L$ is finite in the $\hbar \rightarrow 0$ limit. Thus in the $\hbar \rightarrow 0$ limit the entropy contribution is negligible, compared to that of the energy. 


\section{The variational problem}

Thus, we arrive at the following conclusion:

The quasiclassical tau-function or generalised Seiberg-Witten prepotential is the critical value of the functional

$$
\mathcal{F}(\vec{a}, \mathbf{t})=-\operatorname{Crit}_{f} \mathcal{E}[f]
$$

where we look for the extremum of the energy functional $\mathcal{E}[f]$ in the class $C^{1}(\mathbf{R})$ of differentiable functions $f(x)$, such that

$$
\begin{gathered}
-N \leq f^{\prime}(x) \leq N \\
f^{\prime \prime}(x) \geq 0
\end{gathered}
$$

For $\vec{a}$ in appropriate domain of the moduli space $\mathcal{M}$ the support of $f^{\prime \prime}(x)$ is a set of $n$ disjoint intervals $\left\{\mathbf{I}_{l}\right\}_{l=1, \ldots, n}$ along the real axis:

$$
\begin{gathered}
\operatorname{supp} f^{\prime \prime}=\amalg_{l=1}^{n} \mathbf{I}_{l}, \\
\mathbf{I}_{l}=\left(x_{l}^{-}, x_{l}^{+}\right), \\
x_{1}^{-}<\ldots<x_{l}^{+}<x_{l+1}^{-}<x_{l+1}^{+}<\ldots x_{N}^{+}
\end{gathered}
$$

The moduli $\vec{a}$ enter the variational problem via the additional constraints:

$$
a_{l}=\frac{1}{2} \int_{\mathbf{I}_{l}} d x x f^{\prime \prime}(x)
$$

where $\mathbf{I}_{l}, l=1, \ldots, n$ is the $l$ 'th connected component of $\operatorname{supp} f^{\prime \prime}$.

The number $n$ of cuts, and independent moduli $a_{l}$ depend on the gauge group. For example, for $G=U(N), S U(N), n=N$. For $G=S O(N), n=2\left[\frac{N}{2}\right]$, and for $G=$ $U S p(2 N)$ there are $n=2 N+1$ cuts.

In the $G=S U(N)$ case the Higgs eigenvalues $a_{l}$ obey: $\sum_{l=1}^{N} a_{l}=0$. In the $G=$ $S O(N)$ case the Higgs eigenvalues have the form: $\left(a_{1}, a_{2}, \ldots, a_{n / 2},-a_{1},-a_{2}, \ldots,-a_{n / 2}, *\right)$, 
where for odd $N, *=0$ and for $N$ even $*$ is absent. In the $G=U S p(2 N)$ case the Higgs eigenvalues have the form: $\left(a_{1}, a_{2}, \ldots, a_{N},-a_{1},-a_{2}, \ldots,-a_{N}, 0,0\right)$.

It is easy to notice [29] that our variational problem is very similar to arising in the context of multisupport solutions for matrix integrals. However, there are important distinctions, caused basically by properties of the functions (2.49), which are extremising the functional (2.48). Below we present the solution to the variational problem in the same geometric terms, though involving sometimes the multivalued differentials on hyperelliptic curves. We shall return to the parallels between our approach and the matrix models, when discussing the Eguchi-Yang matrix integral. 


\section{Solution in the $U(1)$ case}

In this chapter we study in detail the extended prepotential in the case of $U(1)$ theory. In the $U(1)$ case, the constraint (2.51) can be in standard way taken into account with the help of the Lagrange multiplier

$$
\mathcal{E}[f] \rightarrow \mathcal{E}[f, a]=\mathcal{E}[f]-a^{D}\left(a-\frac{1}{2} \int d x x f^{\prime \prime}(x)\right)
$$

The Lagrange multiplier $a^{D}$ naively looks like the zeroth time $t_{0}$, since its contribution can be viewed as the shift $\mathbf{t}(x) \rightarrow \mathbf{t}(x)+a^{D} x$. However, in our setup (as often happens for similar problems, see e.g. [30] [31]) the variable $a$ is fixed, while $a^{D}$ is varied, thus the dual variable $a$ plays the role of the zeroth time. For $N>1$ the situation is more complicated and will be discussed later on.

\subsection{The limit shape}

The variational equation for the functional (3.1) gives

$$
-\frac{1}{2} \int d \widetilde{x} f^{\prime \prime}(\widetilde{x})(x-\widetilde{x})(\log |x-\widetilde{x}|-1)+\mathbf{t}^{\prime}(x)=a^{D} \quad x \in \operatorname{supp} f^{\prime \prime}
$$

It is convenient to introduce the following analytic multivalued function

$$
S(z)=-\frac{1}{2} \int d x f^{\prime \prime}(x)(z-x)(\log (z-x)-1)+\mathbf{t}^{\prime}(z)
$$

on the $z$-plane with the supp $f^{\prime \prime}$ removed. It has the following properties:

1.) The differential $\Phi(z)=d S / d z$ is multivalued. However, the differential $d \Phi$ is already well-defined on $\mathcal{C}$, the double cover of the $z$-plane, which is ramified at the end-points of the interval of the support of $f^{\prime \prime}$.

2.) The exponential $\exp (\Re \mathrm{e} \Phi)$ is therefore single-valued on $\mathcal{C}$. On the cut it is equal to one.

3.) The equation (3.2) implies that the real part

$$
\Re \mathrm{e} S(x)=\frac{1}{2}(S(x+i 0)+S(x-i 0))=a^{D}
$$

is constant on the cut $\mathbf{I}=\operatorname{supp} f^{\prime \prime}$. In order to consider the asymptotic of (3.3) in what follows we shall always choose a branch, which is real along the real axis. 
4.) Asymptotically, as $z \rightarrow \infty$,

$$
S(z)=-z(\log z-1)+a \log z+\sum_{k=1}^{\infty} t_{k} z^{k}-\sum_{k=1}^{\infty} \frac{1}{k z^{k}} \frac{\partial \mathcal{F}}{\partial t_{k}}
$$

where, according to (2.48),

$$
\frac{\partial \mathcal{F}}{\partial t_{k}}=\frac{1}{2(k+1)} \int d x x^{k+1} f^{\prime \prime}(x), \quad k>0
$$

and the coefficient in front of the $z(\log z-1)$ term is fixed by

$$
\int d x f^{\prime \prime}(x)=f^{\prime}(+\infty)-f^{\prime}(-\infty)=2
$$

5.) The Legendre transform of $S(z), \Sigma(w)$,

$$
d \Sigma=-z d \Phi=d\left(\mathbf{t}^{\prime}-z \mathbf{t}^{\prime \prime}\right)-\frac{z d z}{2} \int \frac{d x f^{\prime \prime}(x)}{z-x}
$$

expands near $z=\infty, w=\infty$ as follows:

$$
\Sigma(z)=-z-\sum_{k=2}^{\infty}(k-1) t_{k} z^{k}-a \log z+\sum_{k=1}^{\infty} \frac{k+1}{k z^{k}} \frac{\partial \mathcal{F}}{\partial t_{k}}
$$

The formulae (3.5) together with the equation

$$
a^{D}=\frac{\partial \mathcal{F}}{\partial a}
$$

which follows from (2.48), identify the generating function (2.48) with the logarithm of quasiclassical tau-function, being in the case of the single cut, a tau-function of dispersionless Toda chain hierarchy. Note, that the asymptotics (3.4) follows from (2.33)(2.34). Note also that the term, linear in $z$ in (3.8), can be viewed as the regularised $k=1$ term $(k-1) t_{k} z^{k}$, where $t_{1}$ is replaced by the divergent term $\tau_{0}+t_{1}$. The bare coupling $\tau_{0}$ is a logarithmically divergent function of the energy cutoff [3].

6.) The pair of multi-valued functions $(\Phi(z, a), z)$ can be viewed as the quasiclassical analogues of the pair of Orlov-Shulman and Lax operators [22]. The canonical transformation, taking the pair $(\log w, a)$ to $(\Phi, z)$ is generated by the generating function $S(z, a)$, or, equivalently, $\Sigma(\Phi, a)$. 


\subsection{Toda as presentiment}

As we have already discussed, the limit shape is described by the function $f(x)$, which differs from $|x-a|$ on the single interval $\mathbf{I}=\left(x^{-}, x^{+}\right)$. The corresponding integral transforms $S(z)$ and $\Phi(z)$ suggest to study the double cover $\mathcal{C}$ of the $z$-plane with the branch points $z=x^{+}$and $z=x^{-}$.

The curve $\mathcal{C}$ can be equivalently described by the equation

$$
z=v+\Lambda\left(w+\frac{1}{w}\right)
$$

in $\mathbf{C} \times \mathbf{C}^{*}$, with $x^{ \pm}=v \pm 2 \Lambda$. It is just a copy of a Riemann sphere or $\mathbf{C P}^{1}$, with two marked points $P_{ \pm}$, with $z\left(P_{ \pm}\right)=\infty, w^{ \pm 1}\left(P_{ \pm}\right)=\infty$. Another form of the curve (3.10) will be generalised in the nonabelian case:

$$
y^{2}=(z-v)^{2}-4 \Lambda^{2}=\left(z-x^{+}\right)\left(z-x^{-}\right)
$$

where $y=\Lambda\left(w-w^{-1}\right)$. The formula (3.3) defines a function with a logarithmic cut and asymptotic behaviour (3.4), odd under the involution $w \leftrightarrow \frac{1}{w}$ of the curve (3.10). In terms of the variable $w$ one can globally write

$$
S=-S_{E Y}(w)+\sum_{k>0} t_{k} \Omega_{k}(w)+a \log w+a^{D}
$$

with the Eguchi-Yang term

$$
S_{E Y}(w)=\left(v+\Lambda\left(w+\frac{1}{w}\right)\right) \log w+\Lambda(\log \Lambda-1)\left(w-\frac{1}{w}\right),
$$

the "Hamiltonians"

$$
\Omega_{k}(w)=z_{+}^{k}-z_{-}^{k}, \quad k>0
$$

which are the Laurent polynomials in $w$, and the "zeroth Hamiltonian"

$$
\Omega_{0}(w)=\log w
$$

all odd under $w \leftrightarrow \frac{1}{w}$. The Eguchi-Yang term (3.13) is fixed by its asymptotics $S_{E Y}(w)=$ $\pm z(\log z-1)+O(1)$ at $P_{ \pm}($cf. with $(2.37))$. Note that one can write the differentials $d \Omega_{k}$ in the following suggestive form:

$$
d \Omega_{k}=k\left(z^{k-1} y\right)+\frac{d z}{y}
$$


where the symbol $(\ldots)_{+}$denotes now a polynomial part in $z$, in the expansion near $P_{+}$. We can think of (3.12) as of the Legendre transform of the Seiberg-Witten differential $d \Sigma=-z \frac{d w}{w}$. Obviously, on the cut (3.12) gives $\left.S\right|_{w=1}=a^{D}$. The canonical Toda chain times are defined by the residues

$$
t_{0}=\operatorname{res}_{P_{+}} d S=-\operatorname{res}_{P_{-}} d S=a
$$

and

$$
t_{k}=\frac{1}{k} \operatorname{res}_{P_{+}} z^{-k} d S=-\frac{1}{k} \operatorname{res}_{P_{-}} z^{-k} d S, \quad k>0
$$

From the expansion (3.4) at $z \rightarrow \infty$ it also immediately follows, that

$$
\frac{\partial \mathcal{F}}{\partial t_{k}}=\operatorname{res}_{P_{+}} z^{k} d S=-\operatorname{res}_{P_{-}} z^{k} d S, \quad k>0
$$

Notice that (3.4) has no constant in $z$ term, which together with explicit formula (3.12) allows to compute $a^{D}$ from a regularized value of the function (3.12) at $z \rightarrow \infty$

$$
\begin{aligned}
& a^{D}=\left.S\right|_{w=1}= \\
& =\lim _{z \rightarrow \infty}\left(\sum_{k>0} t_{k} z^{k}-z(\log z-1)+a \log z+S_{E Y}(w)-\sum_{k>0} t_{k} \Omega_{k}(w)-a \log w\right)
\end{aligned}
$$

The consistency condition for (3.19) is ensured by the symmetricity of the second derivatives

$$
\frac{\partial^{2} \mathcal{F}}{\partial t_{n} \partial t_{k}}=\operatorname{res}_{P_{+}}\left(z^{k} d \Omega_{n}\right)
$$

where we have introduced

$$
\begin{gathered}
\frac{\partial S}{\partial t_{k}}=\Omega_{k}= \pm\left(z^{k}-\frac{\partial^{2} \mathcal{F}}{\partial a \partial t_{k}}-\sum_{n>0} \frac{\partial^{2} \mathcal{F}}{\partial t_{k} \partial t_{n}} \frac{1}{n z^{n}}\right), \quad k>0 \\
\frac{\partial S}{\partial a}=\Omega_{0}= \pm\left(\log z-\frac{\partial^{2} \mathcal{F}}{\partial a^{2}}-\sum_{n>0} \frac{\partial^{2} \mathcal{F}}{\partial a \partial t_{n}} \frac{1}{n z^{n}}\right)
\end{gathered}
$$

The functions $\Omega_{k}, k>0$, form a basis in the space of meromorphic functions with poles at the points with $P_{ \pm}$, odd under the $w \mapsto w^{-1}$ involution. All time-derivatives here are taken at constant $z$. 
The expansion (3.22) of the Hamiltonian functions (3.14) expresses the second derivatives of $\mathcal{F}$ in terms of the coefficients of the curve (3.10), e.g.

$$
\begin{aligned}
& \Omega_{0}=\log z-\log \Lambda-\frac{v}{z}-\frac{\Lambda^{2}+\frac{v^{2}}{2}}{z^{2}}+\ldots \\
& \Omega_{1}=z-v-\frac{2 \Lambda^{2}}{z}-\frac{2 v \Lambda^{2}}{z^{2}}+\ldots \\
& \Omega_{2}=z^{2}-\left(v^{2}+2 \Lambda^{2}\right)-\frac{4 v \Lambda^{2}}{z}-\frac{2 \Lambda^{2}\left(\Lambda^{2}+2 v^{2}\right)}{z^{2}}+\ldots
\end{aligned}
$$

as $z \rightarrow \infty$, which gives, in particular,

$$
\frac{\partial^{2} \mathcal{F}}{\partial a^{2}}=\log \Lambda, \quad \frac{\partial^{2} \mathcal{F}}{\partial a \partial t_{1}}=v
$$

and

$$
\frac{\partial^{2} \mathcal{F}}{\partial t_{1}^{2}}=2 \Lambda^{2}=2 \exp \left(2 \frac{\partial^{2} \mathcal{F}}{\partial a^{2}}\right)
$$

which becomes the long-wave limit of the Toda chain equations after a derivative with respect to $a$ is taken:

$$
\frac{\partial^{2} a^{D}}{\partial t_{1}^{2}}=2 \frac{\partial}{\partial a} \exp \left(2 \frac{\partial a^{D}}{\partial a}\right)
$$

with the Toda co-ordinate $a^{D}=\frac{\partial \mathcal{F}}{\partial a}$. The other expansion coefficients (3.23), with the help of (3.21), give rise to the Losev-Shatashvili polynomials. In the $U(1)$ case they are polynomials in the single variable $u=v$, whose coefficients depend on $\Lambda$.

One can now find the dependence of the coefficients of the curve (3.10) on the deformation parameters $\mathbf{t}$ of the microscopic theory by computing $d S$ at the ramification points $w= \pm 1$, where $z=v \pm 2 \Lambda$ and $d z=0$ :

$$
\left.\frac{d S}{d \log w}\right|_{w= \pm 1}=\left.\sum_{k>0} t_{k} \frac{d \Omega_{k}}{d \log w}\right|_{w= \pm 1}+a-v \mp 2 \Lambda \log \Lambda=0
$$


Small phase space

If $t_{k}=0$ for $k>1$, solution to (3.27) immediately gives

$$
v=a, \quad \Lambda=e^{t_{1}}
$$

and the prepotential

$$
\mathcal{F}=\frac{1}{2} a a^{D}+\frac{1}{2} \operatorname{res}_{P_{+}}(z d S)-\frac{1}{4} a^{2}=\frac{1}{2}\left(a^{2} t_{1}+e^{2 t_{1}}\right)
$$

The slope $f^{\prime}(x)$ of the corresponding limit shape profile $f(x)$ is given by:

$$
f^{\prime}(x)=\frac{2}{\pi} \arcsin \left(\frac{x-a}{2 e^{t_{1}}}\right), \quad x \in\left(a-2 e^{t_{1}}, a+2 e^{t_{1}}\right)
$$

where the branch of arcsin (hence the name of the "arcsin law" [32]) is chosen so that $f^{\prime}\left(a \pm 2 e^{t_{1}}\right)= \pm 1$. The limit shape itself is given by the function:

$$
f(x)=\frac{2}{\pi}\left(\sqrt{4 e^{2 t_{1}}-(x-a)^{2}}+(x-a) \arcsin \left(\frac{x-a}{2 e^{t_{1}}}\right)\right), \quad x \in\left(a-2 e^{t_{1}}, a+2 e^{t_{1}}\right)
$$

and

$$
f(x)=|x-a|, \quad \text { otherwise }
$$

\section{Turning on $t_{2}$}

Now let us turn on the Casimir $\operatorname{tr} \Phi^{3}$ in the ultraviolet, or in other words consider nonvanishing $t_{1}, t_{2}$. Then one finds from (3.27)

$$
\begin{aligned}
& v=a-\frac{1}{4 t_{2}} \mathbf{L}\left(-16 t_{2}^{2} e^{2\left(t_{1}+2 t_{2} a\right)}\right) \\
& \log \Lambda=t_{1}+2 t_{2} a-\frac{1}{2} \mathbf{L}\left(-16 t_{2}^{2} e^{2\left(t_{1}+2 t_{2} a\right)}\right)
\end{aligned}
$$

where $\mathbf{L}(t)$ has an expansion:

$$
\mathbf{L}(t)=\sum_{n=1}^{\infty} \frac{(-n)^{n-1}}{n !} t^{n}=t-t^{2}+\frac{3}{2} t^{3}-\frac{8}{3} t^{4}+\ldots
$$

and obeys the functional equation

$$
\mathbf{L}(t) \exp \mathbf{L}(t)=t
$$


For the prepotential one gets instead of (3.29)

$$
\begin{aligned}
& \mathcal{F}=\frac{1}{2} a a^{D}+\frac{1}{2} \operatorname{res}_{P_{+}}(z d S)-\frac{t_{2}}{2} \operatorname{res}_{P_{+}}\left(z^{2} d S\right)-\frac{1}{4} a^{2}= \\
& =\mathbf{t}(a)+\frac{1}{2} e^{2 \mathbf{t}^{\prime \prime}(a)}+2 t_{2}^{2} e^{4 \mathbf{t}^{\prime \prime}(a)}+\frac{64 t_{2}^{4}}{3} e^{6 \mathbf{t}^{\prime \prime}(a)}+\frac{1024 t_{2}^{6}}{3} e^{8 \mathbf{t}^{\prime \prime}(a)}+\ldots
\end{aligned}
$$

where the instanton expansion is governed by the parameter $q=e^{\tau_{0}}$ related with the bare coupling, which can be introduced by $t_{1} \rightarrow t_{1}+\log q$. Here $t_{k}=0$ for $k>2$ and $\mathbf{t}(a)=t_{1} \frac{a^{2}}{2}+t_{2} \frac{a^{3}}{3}$, so that $\mathbf{t}^{\prime \prime}(a)=t_{1}+2 t_{2} a$.

The slope of the limit shape is again given by the trigonometric functions:

$$
\begin{aligned}
f^{\prime}(x)= & \frac{2}{\pi}\left(\arcsin \left(\frac{x-v}{2 \Lambda}\right)+2 t_{2} \sqrt{4 \Lambda^{2}-(x-v)^{2}}\right) \\
& v-2 \Lambda \leq x \leq v+2 \Lambda
\end{aligned}
$$

where $v$ and $\Lambda$ are the functions (3.33) of $t_{1}, t_{2}, a$. Hence we see, that the Vershik-Kerov "arcsin" law is deformed, for nonvanishing $t_{2}$, by the Wigner semicircle distribution.

Note that our "rules of the game" are such that the higher order times $t_{k}, k>1$ are considered to be the nilpotent parameters. Had we viewed them as the ordinary perturbation parameters (and thus faced the possible non-renormalisability concerns), the formula (3.37) would have suggested a possibility for the interesting critical behaviour, similar to the one found in the two dimensional Yang-Mills theory [33]: indeed, as soon as $t_{2}$ becomes sufficiently large, $\left|t_{2}\right|>t_{2}^{*}$, so that

$$
4 t_{2}^{*} \Lambda\left(a, t_{1}, t_{2}^{*}\right)=1
$$

the slope $f^{\prime}(x)$ reaches +1 before $x$ reaches the right end of the cut $(v-2 \Lambda, v+2 \Lambda)$. The equation (3.38) is nothing but the convergence radius of the series (3.34) for the function $\mathbf{L}(t)$. For $\left|t_{2}\right|>t_{2}^{*}$ a new cut opens up and the theory goes over to another phase, in particular the curve $\mathcal{C}$ would grow in genus. Of course, the critical value of $t_{2}$ is exponentially large $\sim \Lambda^{-1}$, like the Landau pole.

\subsection{Symplectomorphisms and dispersionless hierarchy}

The evolution of the prepotential $\mathcal{F}(a, \mathbf{t})$ with respect to the times $t_{k}$ is described by the Toda chain hierarchy. We start by reminding the Lax formulation of this hierarchy [22]. The flows can be described in the Hamiltonian fashion, by interpreting the evolution 
to take place on the space of holomorphic functions on $\mathcal{P}=\mathbf{C}^{*} \times \mathbf{C}$. We endow the space $\mathcal{P}$ with the holomorphic symplectic form:

$$
\varpi=\frac{d w}{w} \wedge d a
$$

where $w \in \mathbf{C}^{*}, a \in \mathbf{C}$. The $k^{\prime}$ th flow is governed by the Hamiltonian

$$
\Omega_{k}(w, a)=z_{+}^{k}-z_{-}^{k}, \quad k>1
$$

where, as in $(3.14)(3.10)$ the \pm denote the part, holomorphic at $w=0$ or $w=\infty$, respectively, and $z$ is the Lax operator:

$$
z(w, a)=v(a)+\Lambda(a)\left(w+\frac{1}{w}\right),
$$

and

$$
\Omega_{0}=\log w
$$

The evolution is encoded in the equations:

$$
\begin{aligned}
\frac{\partial}{\partial t_{k}} z(w, a) & =\left\{z(w, a), \Omega_{k}(w, a)\right\} \Leftrightarrow \\
\frac{\partial v(a)}{\partial t_{k}} & +\left(w+\frac{1}{w}\right) \frac{\partial \Lambda(a)}{\partial t_{k}}= \\
& =\Lambda(a)\left(w-\frac{1}{w}\right) \partial_{a} \Omega_{k}-\left(v^{\prime}(a) w+\Lambda^{\prime}(a)\left(w^{2}+1\right)\right) \partial_{w} \Omega_{k}
\end{aligned}
$$

Thus:

$$
\begin{gathered}
\frac{\partial v(a)}{\partial t_{1}}=2\left(\Lambda^{2}(a)\right)^{\prime}, \frac{\partial \Lambda(a)}{\partial t_{1}}=\Lambda(a) v(a)^{\prime} \\
\frac{\partial v(a)}{\partial t_{2}}=4\left(v(a) \Lambda^{2}(a)\right)^{\prime}, \frac{\partial \Lambda(a)}{\partial t_{2}}=\Lambda(a)\left(v(a)^{2}+2 \Lambda^{2}(a)\right)^{\prime}
\end{gathered}
$$

The first line in (3.44) implies the Toda equation (3.25). Let us discuss the geometry behind the equations (3.44)(3.43). They define a set of commuting symplectomorphisms of $\mathcal{P}$. Infinitesimally these symplectomorphisms look like:

$$
\delta w=w \partial_{a} \Omega_{k} \delta t_{k}, \quad \delta a=-w \partial_{w} \Omega_{k} \delta t_{k}
$$

Let us denote the finite time symplectomorphism by:

$$
g(\mathbf{t}): \mathcal{P} \rightarrow \mathcal{P}, \quad(W(w, a ; \mathbf{t}), A(w, a ; \mathbf{t}))=g(\mathbf{t})(w, a)
$$


so that $g(0)=\mathrm{id}$. Now let us take the Lax operator, viewed as a function on $\mathcal{P}$, and let it evolve in the geometric way:

$$
\begin{aligned}
z(w, a ; \mathbf{t})= & g(\mathbf{t})^{*} z(w, a ; 0)=z(W(w, a ; \mathbf{t}), A(w, a ; \mathbf{t}))= \\
& v(A(w, a ; \mathbf{t}))+\Lambda(A(w, a ; \mathbf{t}))\left(W(w, a ; \mathbf{t})+\frac{1}{W(w, a ; \mathbf{t})}\right)= \\
& v(a ; \mathbf{t})+\Lambda(a ; \mathbf{t})\left(w+\frac{1}{w}\right)
\end{aligned}
$$

the last equality being a consequence of the asymptotic behaviour of the Hamiltonian vector fields (3.45) as $w \rightarrow 0$ and $w \rightarrow \infty$.

We can also change the canonical variables, from $(w, a)$ to $(z, \Phi)$, such that:

$$
\frac{d w}{w} \wedge d a=d z \wedge d \Phi
$$

By substituting (3.41) into (3.48) we can express $\Phi$ via $w$ and $a$ (of course, (3.48) determines $\Phi$ up to an addition of a function of $z)$ :

$$
\Phi(w, a)=\int^{a} \frac{d a^{\prime}}{\sqrt{\left(z(w, a)-v\left(a^{\prime}\right)\right)^{2}-4 \Lambda^{2}\left(a^{\prime}\right)}}, \quad z(w, a)=v(a)+\Lambda(a)\left(w+\frac{1}{w}\right)
$$

Finally, the symplectomorphism $g(\mathbf{t})$ can be described with the help of a generating function (see [34] for the definitions):

$$
\mathrm{d} S=\Phi d z+\log w d a
$$

where we view $S$ as a function of $(z, a)$. We can think of $(z, \Phi)$ as of the functions of $W, A$, i.e. they coordinatise the image of $g(\mathbf{t})$. Thus $S$ is a function of $z, a, \mathbf{t}$. We claim it coincides with (3.3). 


\section{The nonabelian case}

\subsection{The energy and surface tension}

In the nonabelian case, $G=U(N), N>1$, similarly to (3.1), one has to add to the functional $\mathcal{E}[f]$ several Lagrange multipliers, or the so-called surface tension term:

$$
\begin{aligned}
& \mathcal{E}[f, \vec{a}]=\mathcal{E}[f]-\sum_{l=1}^{N} a_{l}^{D}\left(a_{l}-\frac{1}{2} \int_{\mathbf{I}_{l}} d x x f^{\prime \prime}(x)\right)= \\
& =\mathcal{E}[f]+\frac{1}{2} a^{D} \int x f^{\prime \prime}(x) d x+\int d x \sigma\left(f^{\prime}\right)-\sum_{l=1}^{N} a_{l} a_{l}^{D}
\end{aligned}
$$

where

$$
a^{D}=\frac{1}{N} \sum_{l=1}^{N} a_{l}^{D}
$$

and $\sigma$ is a piece-wise linear function, which vanishes for $t$ outside the interval $(-N, N)$,

and equals $\frac{1}{2}\left(a_{l}^{D}-a^{D}\right)(t+N-2 l+2)+\sum_{m=1}^{l-1}\left(a_{m}^{D}-a^{D}\right)$, for $t \in(-N+2 l-2,-N+2 l)$, for $l=1, \ldots, N$, so that:

$$
\frac{1}{2} a^{D} \int x f^{\prime \prime}(x) d x+\int d x \sigma\left(f^{\prime}\right)=\frac{1}{2} \sum_{l=1}^{N} a_{l}^{D} \int_{\mathbf{I}_{l}} x f^{\prime \prime}(x) d x
$$

Define, as before:

$$
S(z)=-\frac{1}{2} \int d x f^{\prime \prime}(x)(z-x)(\log (z-x)-1)+\mathbf{t}^{\prime}(z)
$$

and

$$
\Phi(z)=\frac{d S}{d z}=-\frac{1}{2} \int d x f^{\prime \prime}(x) \log (z-x)+\mathbf{t}^{\prime \prime}(z)
$$

\subsection{The curve}

The variational equation, following from (4.1), can be written as:

$$
S(x)=\frac{1}{2}(S(x+i 0)+S(x-i 0))=a_{l}^{D}, \text { for } x \in \mathbf{I}_{l}
$$

which implies:

$$
\Phi(x+i 0)+\Phi(x-i 0)=0, \text { for } x \in \mathbf{I}_{l}
$$


Together with the obvious property

$$
S(x \pm i 0)=a_{l}^{D} \mp \frac{i \pi}{2} \int_{x}^{\infty} d \widetilde{x} f^{\prime \prime}(\widetilde{x})(x-\widetilde{x}), \text { for } x \in \mathbf{I}_{l}
$$

it implies that the differential

$$
d \Phi=S^{\prime \prime}(z) d z=-\frac{1}{2}\left(\int d x \frac{f^{\prime \prime}(x)}{z-x}\right) d z+\mathbf{t}^{\prime \prime \prime} d z
$$

is well-defined on the double cover $\mathcal{C}$ of the $z$-plane, which is ramified at the points $x_{l}^{ \pm}$:

$$
y^{2}=\prod_{l=1}^{N}\left(z-x_{l}^{+}\right)\left(z-x_{l}^{-}\right)
$$

Let us denote by $P_{ \pm}$the two preimages of the point $z=\infty$. The differential $d \Phi$ is odd under the involution $y \mapsto-y$. As $z \rightarrow \infty$, on one of the sheets of $\mathcal{C}$, say near $P_{+}$, the differential $d \Phi$ has the following asymptotics:

$$
d \Phi=\sum_{k>1} k(k-1) t_{k} z^{k-2} d z-N \frac{d z}{z}-a \frac{d z}{z^{2}}-\sum_{k>1} k \frac{\partial \mathcal{F}}{\partial t_{k-1}} \frac{d z}{z^{k+1}}
$$

These two properties allow to write:

$$
d \Phi=\sum_{k \geq 0} s_{k} z^{k} \frac{d z}{y}=\sum_{k>1} k(k-1) t_{k} d \Omega_{k-1}-N d \Omega_{0}-2 \pi i \sum_{j=1}^{N-1} d \omega_{j}
$$

where the differentials $d \Omega_{k}$ are fixed by their asymptotics at $z \rightarrow \infty$

$$
d \Omega_{k} \sim\left\{\begin{array}{c} 
\pm k z^{k-1} d z+O\left(z^{-2}\right), \quad k>0 \\
\pm \frac{d z}{z}+O\left(z^{-2}\right), \quad k=0
\end{array}\right.
$$

and vanishing $A$-periods

$$
\oint_{A_{i}} d \Omega_{k}=0, \quad k \geq 0, \quad \forall i=1, \ldots, N-1
$$

while $d \omega_{i}, i=1, \ldots, N-1$ are canonical holomorphic differentials normalized to the $A$ cycles, surrounding the first $N-1$ cuts.

The coefficients $s_{k}$ and the ramifications points $x_{l}^{ \pm}$are to be determined from the conditions:

$$
\begin{aligned}
& \frac{1}{2 \pi i} \oint_{P_{ \pm}} z^{-k+1} d \Phi= \pm k(k-1) t_{k}, \quad k=2,3, \ldots \\
& \quad \operatorname{res}_{P_{ \pm}} d \Phi=\mp N
\end{aligned}
$$


directly following from (4.11),

$$
\begin{gathered}
-\frac{1}{2 \pi i} \oint_{A_{l}} d \Phi=\frac{1}{2} \int_{\mathbf{I}_{l}} f^{\prime \prime}(x) d x=\frac{1}{2}\left(f^{\prime}\left(x_{l}^{+}\right)-f^{\prime}\left(x_{l}^{-}\right)\right)=1, \\
\frac{1}{2 \pi i} \oint_{B_{l}} d \Phi=0, \quad l=1, \ldots, N
\end{gathered}
$$

If $s(z)=\sum_{k \geq 0} s_{k} z^{k}$ is a polynomial of power $N+K-2$ (in the case of nonvanishing times $t_{1}, \ldots, t_{K}$ up to the $K$-th order), its higher $K$ coefficients are fixed by leading asymptotic and the residue at infinity via (4.14), while the rest $N-1$ coefficients can be determined from fixing the $A$-periods by the upper line in (4.15). Now it leaves only $2 N$ branch points $\left\{x_{j}^{ \pm}\right\}$of the curve (4.10) to be fixed from the rest of (4.15) (vanishing of the $B$-periods), and the Seiberg-Witten periods of the "dual differential"

$$
\frac{1}{2 \pi i} \oint_{A_{l}} d \Sigma=-\frac{1}{2 \pi i} \oint_{A_{l}} z d \Phi=a_{l}, \quad l=1, \ldots, N
$$

Together with normalization of $\Phi\left(x_{N}^{+}\right)=0$ at the largest positive ramification point, suggested by integral representation (4.5), the second conditions of (4.15) can be equivalently written as $2 N$ real conditions

$$
\Re e \Phi\left(x_{j}^{ \pm}\right)=0, \quad j=1, \ldots, 2 N
$$

The condition (4.17) ensure that under variation at constant $z$

$$
\delta(d S)=\delta(\Phi d z) \simeq \text { holomorphic }
$$

modulo the exact terms.

Since under rescaling:

$$
\begin{aligned}
& z \mapsto \mu z, \quad y \mapsto \mu^{N} y \\
& x_{l}^{ \pm} \mapsto \mu x_{l}^{ \pm}, \quad a_{l} \mapsto \mu a_{l}, \quad t_{k} \mapsto \mu^{1-k} t_{k}
\end{aligned}
$$

the function $\Phi(z)$ acquires logarithmic corrections, the $t_{1}$ dependence can be fixed by

$$
t_{1}=\operatorname{res}_{P_{+}}\left(z^{-1} \Phi d z\right)
$$

with the appropriately chosen branch of $\Phi$ at the point $P_{+}$. 
The Lagrange multipliers

$$
a_{l}^{D}=\frac{\partial \mathcal{F}}{\partial a_{l}}, \quad l=1, \ldots, N
$$

can be computed by a standard trick. From (4.6),(4.8) it is clear that

or

$$
a_{i}^{D}-a_{j}^{D}=\int_{x_{j}^{+}}^{x_{i}^{-}} d S=\frac{1}{2} \oint_{B_{i j}} d S
$$

and still

$$
\frac{\partial \mathcal{F}}{\partial a_{i}}=\frac{1}{2} \oint_{B_{i}} d S, \quad i=1, \ldots, N-1
$$

$$
a^{D}=\frac{1}{N} \sum_{i=1}^{N} a_{i}^{D}=\frac{1}{N} \sum_{i=1}^{N} \frac{\partial \mathcal{F}}{\partial a_{i}}
$$

For the other time-derivatives one can write

$$
\frac{\partial \mathcal{F}}{\partial t_{k}}=\operatorname{res}_{P_{+}}\left(z^{k} d S\right)=-\frac{1}{k+1} \operatorname{res}_{P_{+}}\left(z^{k+1} d \Phi\right)
$$

\subsection{Small phase space and Seiberg-Witten curves}

Now suppose:

$$
\mathbf{t}(x)=t_{1} \frac{x^{2}}{2},
$$

The conditions (4.11)(4.15) imply that $w=\exp (-\Phi)$ is a meromorphic function on $\mathcal{C}$ with the only pole at $P_{+}$, where it behaves as:

$$
w \propto z^{N}+\ldots,
$$

(recall that $z\left(P_{+}\right)=\infty$ ) and which transforms under the $y \mapsto-y$ involution as $w \mapsto w^{-1}$, (for the switched on higher times, such function would acquire an essential singularity at the points where $z=\infty$, and the constraints (4.15) cannot be resolved algebraically).

These conditions fix $w$ uniquely, up to a few parameters:

$$
\Lambda^{N}\left(w+\frac{1}{w}\right)=\prod_{l=1}^{N}\left(z-v_{l}\right) \equiv P_{N}(z)
$$

i.e.

$$
d \Phi=-P_{N}^{\prime}(z) \frac{d z}{y}, \quad y=\Lambda^{N}\left(w-\frac{1}{w}\right)
$$

The coefficients of $P_{N}(z)$ are determined by the periods (4.15):

$$
-\frac{1}{2 \pi i} \oint_{A_{l}} z d \Phi=a_{l}, l=1, \ldots, N
$$

Finally, the parameter $\Lambda$ is related to the time $t_{1}$ via:

$$
\Lambda^{N}=e^{t_{1}}
$$

In this way one easily recovers the results of [3]. 


\subsection{Quasiclassical flows}

Let us now come back to the general formula (1.7) and show how it can be derived from our quasiclassical solution on large phase space, or for nonvanishing microscopic times. Taking an extra derivative of (4.22) one gets formula (3.21), which can be re-written on generic non-abelian curve (4.10) as

$$
\frac{\partial^{2} \mathcal{F}}{\partial t_{n} \partial t_{m}}=\operatorname{res}_{P_{+}}\left(z^{m} d \Omega_{n}\right)=\operatorname{res}_{P_{+} \otimes P_{+}}\left(z(P)^{n} z\left(P^{\prime}\right)^{m} W\left(P, P^{\prime}\right)\right)
$$

where we have introduced the bi-differential $W\left(P, P^{\prime}\right)=d_{P} d_{P^{\prime}} \log E\left(P, P^{\prime}\right), E\left(P, P^{\prime}\right)$ being the prime form [35], with the only second order pole at diagonal and vanishing $A$ periods. In the inverse co-ordinates $z=z(P)$ and $z^{\prime}=z\left(P^{\prime}\right)$ near the point $P^{+}$with $z\left(P^{+}\right)=\infty$ it has expansion

$$
W\left(z, z^{\prime}\right)=\frac{d z d z^{\prime}}{\left(z-z^{\prime}\right)^{2}}+\ldots=\sum_{k>0} \frac{d z}{z^{k+1}} d \Omega_{k}\left(z^{\prime}\right)+\ldots
$$

The bi-differential $W\left(P, P^{\prime}\right)$ can be related with the Szegö kernel [35]

$$
S_{e}\left(P, P^{\prime}\right) S_{-e}\left(P, P^{\prime}\right)=W\left(P, P^{\prime}\right)+d \omega_{i}(P) d \omega_{j}\left(P^{\prime}\right) \frac{\partial}{\partial \tau_{i j}} \log \vartheta_{e}(0 \mid \tau)
$$

which, for a half-integer characteristics $e \equiv-e$, has an explicit expression on hyperelliptic curve (4.10)

$$
S_{e}\left(z, z^{\prime}\right)=\frac{U_{e}(z)+U_{e}\left(z^{\prime}\right)}{2 \sqrt{U_{e}(z) U_{e}\left(z^{\prime}\right)}} \frac{\sqrt{d z d z^{\prime}}}{z-z^{\prime}}
$$

with

$$
U_{e}(z)=\prod_{j=1}^{N} \sqrt{\frac{z-x_{e_{j}^{+}}}{z-x_{e_{j}^{-}}}}
$$

Here $\left\{x_{e_{j}^{ \pm}}\right\}$is a partition of the ramification points of (4.10) into two sets, corresponding to a given characteristic $e$. For example, on a small phase space, when (4.10) turns into the Seiberg-Witten curve (4.24), there is a distinguished partition $e=E$ with

$$
U_{E}(z)=\sqrt{\frac{P_{N}(z)-2 \Lambda^{N}}{P_{N}(z)+2 \Lambda^{N}}}
$$


Substitution of (4.28) into (4.27) gives

$$
\begin{aligned}
& \frac{\partial^{2} \mathcal{F}}{\partial t_{n} \partial t_{m}}=\operatorname{res}_{P_{+} \otimes P_{+}}\left(z(P)^{n} z\left(P^{\prime}\right)^{m} S_{e}\left(P, P^{\prime}\right)^{2}\right)- \\
& -\operatorname{res}_{P_{+}}\left(z^{n} d \omega_{i}\right) \operatorname{res}_{P_{+}}\left(z^{m} d \omega_{j}\right) \frac{\partial}{\partial \tau_{i j}} \log \vartheta_{e}(0 \mid \tau)= \\
& =\mathcal{C}_{n m}\left(x_{e_{j}^{ \pm}}\right)-\frac{\partial^{2} \mathcal{F}}{\partial a_{i} \partial t_{n}} \frac{\partial^{2} \mathcal{F}}{\partial a_{j} \partial t_{m}} \frac{\partial}{\partial \tau_{i j}} \log \vartheta_{e}(0 \mid \tau)
\end{aligned}
$$

where for the Losev-Shatashvili polynomials one gets from (4.29)

$$
\mathcal{C}_{n m}\left(x_{e_{j}^{ \pm}}\right)=\frac{1}{2} \operatorname{res}_{P_{+} \otimes P_{+}}\left(\frac{z^{k} z^{\prime n}}{\left(z-z^{\prime}\right)^{2}}\left(1+\frac{U_{e}(z)}{2 U_{e}\left(z^{\prime}\right)}+\frac{U_{e}\left(z^{\prime}\right)}{2 U_{e}(z)}\right) d z d z^{\prime}\right)
$$

If calculated on the small phase space, where all $t_{k}=0$, with $k>1$, and the particular choice of the characteristic (4.31), which correspond to the deformation (1.7) around the Seiberg-Witten prepotential, residues in (4.33) vanish for $n, m<N$, and one gets

$$
\frac{\partial^{2} \mathcal{F}}{\partial t_{n} \partial t_{m}}=-\frac{\partial u_{n+1}}{\partial a_{i}} \frac{\partial u_{m+1}}{\partial a_{j}} \frac{\partial}{\partial \tau_{i j}} \log \vartheta_{E}(0 \mid \tau), \quad n, m<N
$$

with

$$
u_{n}=\frac{\partial \mathcal{F}}{\partial t_{n-1}}=\frac{1}{n} \operatorname{res}_{P_{+}}\left(z^{n} \frac{P_{N}^{\prime} d z}{y}\right)=\frac{1}{n} \sum_{l=1}^{N} v_{l}^{n}=\frac{1}{n}\left\langle\operatorname{tr} \Phi^{n}\right\rangle
$$

justifying (1.7).

Our derivation of the renormalisation group equation almost repeats, or even simplifies, the derivation from [8]. The main difference is that the formalism of quasiclassical hierarchy is developed now at fixed hyperelliptic co-ordinate $z$, while in [8] the role of such distinguished co-ordinate was played by the co-ordinate on base torus $w$. In the last case the quasiclassical flows were not deforming the geometry of the Seiberg-Witten curves (only the generating differential had to be replaced), but the Losev-Shatashvili polynomials (the analogs of (4.33)) were never vanishing, being instead related to the generalised Kontsevich model, or topological Landau-Ginzburg models [36]. Now we see, that algebraic $w$ has to be replaced by the transcendental $\Phi$. The proper choice of local co-ordinate $z$ is suggested by the microscopic theory, where it is already encoded in $\mathcal{F}_{\mathrm{UV}}=\operatorname{tr} \mathbf{t}(\Phi)$, see (1.6), and therefore it appears to be distinguished in the effective functional (2.48). We can conclude therefore, that the quasiclassical hierarchies arise as adequate language for the effective low-energy theories, but to specify the details (the choice of proper basis, local co-ordinates etc) one needs to turn directly to a microscopic theory. 


\subsection{Instanton expansion with higher times}

Let us now test our results in the non-abelian case against explicit calculations of the first instanton corrections to the prepotential. We shall see immediately that extracting even the first term in the instanton expansion of the Krichever tau-function is a nontrivial task, and the direct evaluation using the sum over partitions here is much more effective. The true application of the quasiclassical tau-function is the analysis of the nonperturbative effects such as the emergence of the massless BPS particles at some points of the moduli space of vacua, the surfaces of marginal stability and so on. However, when we study the extended Seiberg-Witten theory, the "new" directions coming from the "times" $t_{k}$ which couple to the higher Casimirs, are to be viewed as nilpotent (much like the generalised moduli space of the topological string of B type on Calabi-Yau manifold [37]). We should not attempt to give a physical meaning to the singularities which might occur at finite values of $t_{k}$ 's. Note that adding matter to the $\mathcal{N}=2$ theory effectively switches on the times $t_{k}$. For example, if we add $N_{f}$ fundamental multiplets, we get $t_{k} \sim \frac{1}{k-1} \sum_{f} m_{f}^{-2 k}$. In the theory with "real" fundamental multiplets the prepotential has singularities when the charged matter becomes massless, i.e. near $a \sim m_{f}$, which is near infinity for small $t$.

Let us start with the perturbative limit with the higher times switched on. It is characterized by the degenerate differential (4.12)

$$
d \Phi_{0}=\mathbf{t}^{\prime \prime \prime}(z) d z-\sum_{l=1}^{N} \frac{d z}{z-v_{l}}
$$

where the position of poles of degenerate holomorphic differentials coincide with the perturbative values of the Seiberg-Witten periods

$$
a_{l}=-\operatorname{res}_{v_{l}}\left(z d \Phi_{0}\right)=v_{l}, \quad l=1, \ldots, N
$$

Integrating (4.36),

$$
\Phi_{0}=\mathbf{t}^{\prime \prime}(z)-\sum_{l=1}^{N} \log \left(z-a_{l}\right)
$$

one gets the perturbative generating differential $d S_{0}=\Phi_{0} d z$, satisfying

$$
\frac{\partial d S_{0}}{\partial a_{l}}=\frac{d z}{z-a_{l}}, \quad l=1, \ldots, N
$$




$$
\frac{\partial d S_{0}}{\partial t_{k}}=k z^{k-1} d z, \quad k>0
$$

and, in the rational case, it is easy to write the function

$$
S_{0}(x)=\mathbf{t}^{\prime}(x)-\sum_{l=1}^{N}\left(x-a_{l}\right)\left(\log \left(x-a_{l}\right)-1\right)
$$

Equations

$$
a_{j}^{D}=\frac{\partial \mathcal{F}_{0}}{\partial a_{j}}=S_{0}\left(a_{j}\right)
$$

define the perturbative prepotential with switched on higher Casimirs to be

$$
\mathcal{F}_{0}=\sum_{l=1}^{N} \mathbf{t}\left(a_{l}\right)+\frac{1}{2} \sum_{m \neq l} \mathbf{F}\left(a_{l}-a_{m}\right)
$$

to be the sum of the ultraviolet prepotential $\mathcal{F}_{\mathrm{UV}}=\operatorname{tr} \mathbf{t}(\Phi)$ and the perturbative $\mathcal{N}=2$ prepotential, with the "Seiberg-Witten" function defined in (2.15).

Consider now vanishing $B$-period (4.15) of the perturbative differential (4.36)

$$
\int_{x_{i}^{+}}^{x_{j}^{-}} d \Phi_{0}=0
$$

where $x_{j}^{ \pm}=a_{j} \pm 2 \sqrt{q S_{j}}+O\left(q^{2}\right)$ are positions of the branching points of the curve (4.10) in the vicinity of perturbative rational curve. Equation $\Phi_{0}\left(x_{i}^{+}\right)=\Phi_{0}\left(x_{j}^{-}\right)$in the first nonvanishing order in parameter of instanton expansion $q$ gives

$$
S_{l}=\frac{e^{2 \mathbf{t}^{\prime \prime}\left(a_{l}\right)}}{\prod_{m \neq l}\left(a_{l}-a_{m}\right)^{2}}, \quad l=1, \ldots, N
$$

where the numeric coefficient is fixed, say, from comparison with the Seiberg-Witten curve (4.24) on a small phase space.

Take now for simplicity the $U(2)$ gauge group. Then for the equation of the curve (4.10) up to the second order in $q$ one has

$$
\begin{aligned}
& y^{2}=\prod_{l=1,2}\left(\left(z-a_{l}\right)^{2}-4 q S_{l}+\ldots\right)= \\
& =\left(z-a_{1}\right)^{2}\left(z-a_{2}\right)^{2}-4 q\left(S_{1}\left(z-a_{2}\right)^{2}+S_{2}\left(z-a_{1}\right)^{2}\right)+O\left(q^{2}\right)
\end{aligned}
$$

and

$$
\frac{d z}{y}=\frac{d z}{\left(z-a_{1}\right)\left(z-a_{2}\right)}\left(1+2 q\left(\frac{S_{1}}{\left(z-a_{1}\right)^{2}}+\frac{S_{2}}{\left(z-a_{2}\right)^{2}}\right)\right)+O\left(q^{2}\right)
$$


One can also expand therefore (4.12) as $d \Phi=d \Phi_{0}+q d \Phi_{1}+O\left(q^{2}\right)$ with

$$
\begin{aligned}
& d \Phi_{1}=2\left(\mathbf{t}^{\prime \prime \prime}(z)-\frac{2 z-a_{1}-a_{2}}{\left(z-a_{1}\right)\left(z-a_{2}\right)}\right)\left(\frac{S_{1}}{\left(z-a_{1}\right)^{2}}+\frac{S_{2}}{\left(z-a_{2}\right)^{2}}\right)- \\
& -\frac{2\left(S_{1}-S_{2}\right)}{\left(a_{1}-a_{2}\right)\left(z-a_{1}\right)\left(z-a_{2}\right)}
\end{aligned}
$$

normalized to $\operatorname{res}_{a_{1}} d \Phi_{1}=\operatorname{res}_{a_{2}} d \Phi_{1}=0$ (we restrict ourselves now for $\mathbf{t}^{\prime \prime \prime}(z)=2 t_{2}$ with only nonvanishing $\left.t_{1}, t_{2} \neq 0\right)$. Computation of residues of (4.43) at infinity gives

$$
\begin{aligned}
& -\frac{1}{2} \operatorname{res}_{\infty}\left(z^{2} d \Phi_{1}\right)=a_{1} \delta a_{1}+a_{2} \delta a_{2}+S_{1}+S_{2} \\
& -\frac{1}{3} \operatorname{res}_{\infty}\left(z^{3} d \Phi_{1}\right)=a_{1}^{2} \delta a_{1}+a_{2}^{2} \delta a_{2}+2\left(a_{1} S_{1}+a_{2} S_{2}\right)
\end{aligned}
$$

Taking into account that (4.22) gives at linear order in $q$

$$
-\frac{1}{k+1} \operatorname{res}_{\infty}\left(z^{k+1} d \Phi_{1}\right)=\left.\frac{\partial \mathcal{F}}{\partial t_{k}}\right|_{q}=\sum_{l=1}^{N} a_{l}^{k-1} \delta a_{l}+\frac{\partial \mathcal{F}_{1}}{\partial t_{k}}
$$

where the first term in the r.h.s. arises as a contribution from the perturbative prepotential (4.40) due to instantonic renormalization of the relation between the coefficients of the curve (4.10) and the Seiberg-Witten periods as $a_{i} \rightarrow a_{i}+q \delta a_{i}$ with

$$
\begin{aligned}
& \delta a_{1}=\left(2 t_{2}-\frac{1}{a_{1}-a_{2}}\right) S_{1}=\operatorname{res}_{a_{1}}\left(z d \Phi_{1}\right) \\
& \delta a_{2}=\left(2 t_{2}+\frac{1}{a_{1}-a_{2}}\right) S_{2}=\operatorname{res}_{a_{2}}\left(z d \Phi_{1}\right)
\end{aligned}
$$

Comparing (4.45) with (4.44) and taking into account (4.46), one gets $\mathcal{F}_{1}=\frac{1}{2} \sum_{l} S_{l}$, what coincides with direct instantonic calculus. Higher coefficients of the instantonic expansion of the quasiclassical tau-function can be obtained in a similar way. 


\section{Eguchi-Yang picture}

In 1994, T. Eguchi and S. Yang have proposed [17] the following matrix model:

$$
Z_{N}(t)=\int \mathcal{D} M \exp N \operatorname{tr}\left(-2 M(\log M-1)+\sum_{k>0} t_{k} M^{k}\right)
$$

for the description of the type $A \mathbf{C P}^{1}$ topological string, in the stationary sector of GromovWitten theory (some generalisations for other target spaces were studied in [38]). Their proposal is more general, it includes the full phase space of the sigma model, i.e. the full set of gravitational descendants of the cohomology of $\mathbf{C P}^{1}$.

One can easily relate the matrix model (5.1) to our variational problem (3.2). We shall show that the proposal [17] is correct as far as the genus zero part of the topological string is concerned, if one views (5.1) as the integral over the supermatrices of $\left(N_{+} \mid N_{-}\right) \times\left(N_{+} \mid N_{-}\right)$ size.

Indeed, let us study the saddle point approximation to the matrix integral (5.1), in the large $N$ limit. As usual, we assume the eigen-values to form a distribution, with the continuous density of the eigenvalues:

$$
\rho(x)=\frac{1}{N}\langle\operatorname{tr} \delta(x-M)\rangle
$$

The action of the matrix model (5.1) together with the contribution of the measure (the Vandermonde determinant) combine together nicely to the following effective action functional of $\rho(x)$ :

$\mathcal{V}_{\text {eff }}[\rho]=2 \int_{x_{1}>x_{2}} d x_{1} d x_{2} \rho\left(x_{1}\right) \rho\left(x_{2}\right) \log \left(x_{1}-x_{2}\right)+\int d x \rho(x)\left(-2 x(\log (x)-1)+\sum_{k>0} t_{k} x^{k}\right)$

In the case of bosonic matrix model the density function $\rho(x)$ is a non-negative function on the real line which has a compact support. The obvious constraint for (5.2)

$$
\int d x \rho(x)=1
$$

can be enforced with the help of a Lagrange multiplier:

$$
\mathcal{V}_{\text {eff }}[\rho] \rightarrow \mathcal{V}_{\text {eff }}[\rho]-a^{D}\left(\int d x \rho(x)-1\right)
$$


The saddle point equation reads now:

$$
2 \int d \widetilde{x} \rho(\widetilde{x}) \log |x-\widetilde{x}|-2 x(\log (x)-1)+\sum_{k>0} t_{k} x^{k}=a^{D}, \quad x \in \operatorname{supp} \rho
$$

Note that once we shift $x \rightarrow x-a$, make an identification:

$$
f^{\prime}(x)=\operatorname{sgn}(x)-\rho(x)
$$

and rescale the times $t_{k}$ and $a^{D}$ by an irrelevant here factor of $2^{\ddagger}$, the equation (5.5) becomes equivalent to (3.2). It can be shown by integrating (3.2) by parts and choosing the real branch of the logarithm function. However, the "density" $\rho(x)$ which corresponds to the saddle point of (3.1) is not non-negative. For example, on the small phase space, for $a=0$,

$$
\rho(x)=\operatorname{sgn}(x)-\frac{2}{\pi} \arcsin \left(\frac{x}{2 e^{t_{1}}}\right)
$$

which has a jump at $x=0$, where it goes from being positive to being negative. Note also that for (5.7) obviously:

$$
\int d x \rho(x)=0
$$

(which is also consistent with (5.6) and (2.51)).

What could this negative density of eigenvalues possibly mean? The hint comes from (5.8). Since the integral of the density of eigenvalues is proportional to the trace of unit matrix, one can conclude that the "matrix model" corresponding to the original model of random partitions (1.8) must have vanishing trace of the unit matrix. The natural venue for such matrices is the supermatrix model (see, e.g. [39]), where the traces are replaced by supertraces. For the $\left(N_{+} \mid N_{-}\right) \times\left(N_{+} \mid N_{-}\right)$matrices the supertrace of a unit operator is equal to $N_{+}-N_{-}$, which can be both positive and negative.

$$
M=\left(\begin{array}{cc}
A & B \\
C & D
\end{array}\right), \quad \operatorname{str} M=\operatorname{tr} A-\operatorname{tr} D
$$

where $A$ and $D$ are $N_{+} \times N_{+}$and $N_{-} \times N_{-}$size hermitian matrices with bosonic entries, respectively; $B$ and $C$ are fermion-valued $N_{+} \times N_{-}$and $N_{-} \times N_{+}$dimensional complex

$\ddagger$ The factor of 2 in (5.1) in front of the Eguchi-Yang term is a consequence of the fact that matrix integral is rather related to the correlation function bilinear in fermions, cf. e.g. with [19]. In particular, this is related to the fact that integration measure contains the square of the Vandermonde determinant. 
matrices, $B=C^{\dagger}$. The only subtlety in the definition of the measure $\mathcal{D} M$ is the division by the volume of the gauge group, which is done by the proper treatment of ghosts (both bosonic and fermionic). The invariance of the measure and the action (which is the same as (5.1) with the replacement $N \operatorname{tr} \rightarrow \frac{1}{h} \operatorname{str}$, with the new "supermatrix Planck constant" $h$ ) is such that both $A$ and $D$ can be diagonalised:

$$
A \rightarrow \operatorname{diag}\left(x_{1}^{+}, \ldots, x_{N_{+}}^{+}\right), \quad D \rightarrow \operatorname{diag}\left(x_{1}^{-}, \ldots, x_{N_{-}}^{-}\right)
$$

We can view (5.10) as a gauge-fixing condition. The corresponding Fadeev-Popov determinant equals (note again its similarity with the (square of the) free fermion correlator):

$$
\left(\frac{\prod_{1 \leq i<j \leq N_{+}}\left(x_{i}^{+}-x_{j}^{+}\right) \prod_{1 \leq i<j \leq N_{-}}\left(x_{i}^{-}-x_{j}^{-}\right)}{\prod_{1 \leq i \leq N_{+}} \prod_{1 \leq j \leq N_{-}}\left(x_{i}^{+}-x_{j}^{-}\right)}\right)^{2}
$$

Now one can write for the density of eigenvalues (5.10):

$$
\rho(x)=h\left(\sum_{i=1}^{N_{+}} \delta\left(x-x_{i}^{+}\right)-\sum_{i=1}^{N_{-}} \delta\left(x-x_{i}^{-}\right)\right)
$$

and consider it in the large $N$ limit, i.e. when $h \rightarrow 0$ as $N_{ \pm} \rightarrow \infty$, so that $h N_{ \pm}$are finite. The logarithm of jacobian (5.11) plus the Eguchi-Yang action are still given by $\frac{1}{h^{2}} \mathcal{V}_{\text {eff }}[\rho]$, defined in (5.3), but the supermatrix density (5.12) is automatically subjected to (5.8) upon $N_{+}=N_{-}$, which can be re-expressed in terms of times of the Toda hierarchy. For example, on the small phase space:

$$
h N_{ \pm} \rightarrow 2 \frac{\pi-2}{\pi} e^{t_{1}}
$$

while in general:

$$
h N_{ \pm} \rightarrow\left|x^{ \pm}(\mathbf{t})-a\right|
$$

It would be nice to investigate our supermatrix model further, perhaps extending the saddle point correspondence to the exact relation between the $\hbar$ and $\frac{1}{N_{ \pm}}$(or $h$ )-expansions. Perhaps it is worthwhile to study more general supermatrix models, with $N_{+} \neq N_{-}$. It is also interesting to find out whether there is a relation of our supermatrix model and the Dijkgraaf-Vafa matrix models, computing the superpotentials of $\mathcal{N}=1$ supersymmetric gauge theories, as suggested in [1][3]. In this paper we have pointed out new features of the matrix models possibly relevant for the $\mathcal{N}=2$ physics: the non-positivity of eigenvalue densities, which could be related to the block-matrix decompositions arising in the perturbative matrix model calculations (cf. e.g. with [40],[41] where such decomposition was performed for bosonic one- and two- matrix models). 


\section{Conclusions and future directions}

In this paper we have solved the long-standing problem of extending the prepotential of the effective $\mathcal{N}=2$ theory to the "large phase space", obtained by turning on the chiral perturbations

$$
\Delta \mathcal{L}=\int d^{4} \theta \mathbf{t}(\mathbf{\Phi})
$$

where $\mathbf{t}$ is a gauge-invariant function. We have seen that for the single-trace perturbations the resulting prepotential coincides with the Krichever tau-function of the quasiclassical hierarchy. For the $U(1)$ theory this is the dispersionless Toda chain hierarchy. For the $U(N)$ theory this more general quasiclassical or universal Whitham hierarchy (in the sense of $[6])$ of the Toda-chain type (i.e. it corresponds to hyperelliptic curve with two marked points $\left.P_{ \pm}\right)$.

The derivation of the paper was based on a particular identification of the operators (6.1) with some cohomology classes of the moduli spaces $\mathcal{M}_{k}$ of gauge instantons, and their continuation on the partial compactifications $\overline{\mathcal{M}}_{k}$. For the gauge theory with gauge group $U(N)$ the compactification $\overline{\mathcal{M}}_{k}$ is the moduli space of rank $N$ torsion free sheaves on $X=\mathbf{C P}^{2}$ with the fixed trivialisation at the fixed complex line $D=\mathbf{C P}_{\infty}^{1}$ (this is also a moduli space of noncommutative instantons [9]). The operator (6.1) of the form:

$$
\int d^{4} \theta \operatorname{tr} \boldsymbol{\Phi}^{k}
$$

is identified with the $k$-th component of the Chern character $\operatorname{ch}(\mathcal{E})$ of the universal sheaf

$$
\mathcal{E} \rightarrow \overline{\mathcal{M}}_{k} \times X
$$

One can imagine other definitions of the ultraviolet observables (6.1). This discussion is parallel to the discussion of the 2-observables in the two dimensional sigma models [10]. The integrable structure which we find with our prescription is very suggestive. Of course, the arguments [42] which show that the moduli space of vacua of $\mathcal{N}=2$ supersymmetric gauge theory is a base of an algebraic integrable system, based on electric-magnetic duality, do not apply per se to the large phase space, spanned by the couplings $t_{k}$. However, one can think, at least formally, of them as coming from the degeneration of the standard moduli space of vacua of the theory with larger gauge group, more matter and so on.

The appearance of quasiclassical hierarchies in the Seiberg-Witten theory was anticipated long time ago [4]. The program of studying the "large phase space" of the SeibergWitten theory was put forward in [7] and some proposals about corresponding integrable 
hierarchies were made in [4][8][43]. The subtlety of all these proposals is the contact term ambiguity. In a sense it is a standard problem of reconstructing the ultraviolet theory given the infrared one. We have shown in this paper, that the quasiclassical hierarchy, in the basis of time-variables proposed by microscopic instanton theory, solves the ambiguity problem exactly in the way anticipated in [7]. For small $k, l$ the Losev-Shatashvili polynomials can be also studied using the blowup techniques [7][44].

One should keep in mind the richness of physics which is related to the random partition models of which we studied the thermodynamic limits: our results can be easily extended to the five dimensional supersymmetric gauge theories (which means going beyond the small phase space analysis of [23]) where they become the statements about the dispersionless hierarchies governing the melting of the crystals with appropriate symmetries (thus generalizing the work of [45]); the same fermion correlators and their dispersionless limits show up in the physics of one dimensional electrons [46] ("physics of quantum wires"). Finally, one may study more seriously the analogues of Douglas-Kazakov phase transition on the large phase space, and also incorporate the multi-trace ultraviolet perturbations. We hope to return to this discussion elsewhere.

\section{Acknowledgements.}

We are grateful to Sergei Kharchev, Igor Krichever, Andrei Losev and Andrei Okounkov for numerous discussions.

The work of AM was partially supported by Russian Ministry of Industry, Science and Technology under the contract 40.052.1.1.1112, by the grants РФФИ 05-02-17451, HШ-4401.2006.2 and INTAS 05-1000008-7865, by the grant ANR-05-BLAN-0029-01 "Geometry and integrability in mathematical physics", and by the NWO-РФФИ program 047.017.2004.015 "Geometric Aspects of Quantum Theory and Integrable Systems". The research of NN was partially supported by European RTN under the contract 005104 "ForcesUniverse", by ANR under the grants ANR-06-BLAN-3_137168 "Structure of vacuum, topological strings and black holes", and ANR-05-BLAN-0029-01 "Geometry and integrability in mathematical physics", and by the grants РФФИ 06-02-17382 and НШ8065.2006.2. AM thanks Max-Planck-Institut für Mathematik, Bonn; Institut des Hautes Etudes Scientifiques, Bures-sur-Yvette, and Laboratoire de Mathématiques et Physique Théorique de l'Université de Tours, while NN thanks the Institute for Advanced Study at Princeton, MSRI, Department of Mathematics at UC Berkeley and the New High Energy Theory Center at Rutgers University, for hospitality during various stages of preparation of the manuscript. 


\section{Appendix A. Partitions and Chern characters}

Partitions and Young diagrams (the standard reference is [24]).

For each partition $\lambda(2.4)$, its size $|\lambda|=\lambda_{1}+\lambda_{2}+\ldots+\lambda_{\ell_{\lambda}}$ is a number of boxes in the so-called Young diagram $Y(\lambda)$, which is a geometric way to represent partition. The length $\ell_{\lambda}$ is the number of columns. Traditionally one draws Young diagrams as the collection of rows of boxes, of the lengths $\lambda_{1}, \lambda_{2}$, etc. Here we draw an example of the Young diagram, for the partition $\lambda=(5,2)$ with $\ell_{\lambda}=2,|\lambda|=7$ :

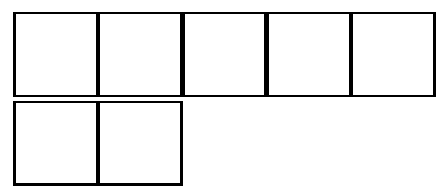

and its profile function $f_{\lambda}(x)$ defined in (2.5),

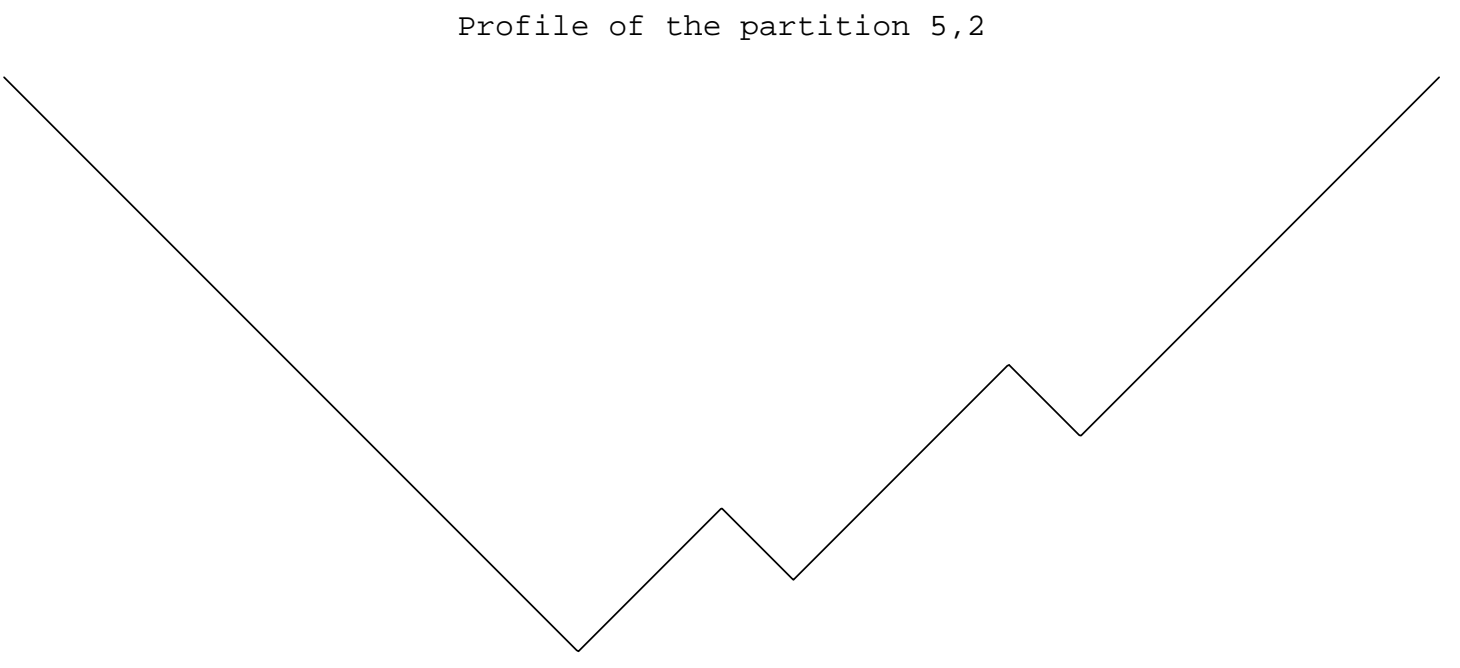

Fig.1

For partition $\lambda$ one introduces the dual partition $\lambda^{\prime}$, whose Young diagram $Y\left(\lambda^{\prime}\right)$ is obtained by exchanging the rows and columns of $Y(\lambda)$. For example, the partition, 
dual to $(5,2)$,

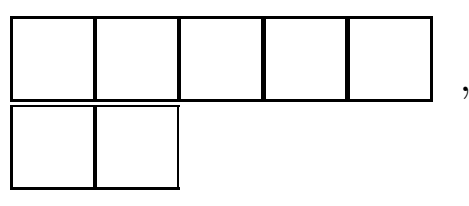

is the partition

$(2,2,1,1,1)$ :

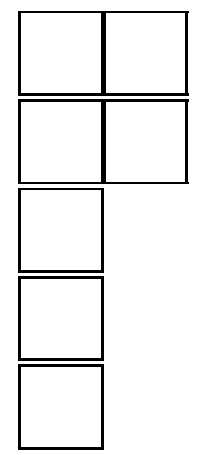

It is convenient to introduce a coordinate system, $(i, j)$, on the Young diagram:

$$
\begin{aligned}
& 1 \leq j \leq \lambda_{i} \leq \ell_{\lambda^{\prime}} \\
& 1 \leq i \leq \lambda_{j}^{\prime} \leq \ell_{\lambda}
\end{aligned}
$$

The coordinate $i$ labels rows, from top to bottom, while the coordinate $j$ labels columns, from left to right. For example, the partition $\lambda=(5,2)$ gets coordinatised in the following way:

\begin{tabular}{|l|l|l|l|l|}
\hline$(1,1)$ & $(1,2)$ & $(1,3)$ & $(1,4)$ & $(1,5)$ \\
\hline \hline$(2,1)$ & $(2,2)$ & \\
\hline
\end{tabular}

For the box with the coordinates $(i, j)$, the hook-length is defined as: $h_{(i, j)}=\lambda_{i}+\lambda_{j}^{\prime}-i-$ $j+1$. In our example $\lambda=(5,2)$ :

\begin{tabular}{|l|l|l|l|l|}
\hline$h_{(1,1)}=6$ & $h_{(1,2)}=5$ & $h_{(1,3)}=3$ & $h_{(1,4)}=2$ & $h_{(1,5)}=1$ \\
\hline \hline$h_{(2,1)}=2$ & $h_{(2,2)}=1$ & \multicolumn{2}{l}{} \\
\hline
\end{tabular}

Finally, the Plancherel measure, which appears in $(1.8),(2.11)$ is just the product of the hook-length's

$$
\mathbf{m}_{\lambda}=\prod_{\square \in \lambda} \frac{1}{h_{\square}}=\prod_{i=1}^{\ell_{\lambda}} \frac{\left(\ell_{\lambda}-i\right) !}{\left(\ell_{\lambda}+\lambda_{i}-i\right) !} \prod_{1 \leq i<j \leq \ell_{\lambda}} \frac{\lambda_{i}-\lambda_{j}+j-i}{j-i}=\prod_{i<j} \frac{\lambda_{i}-\lambda_{j}+j-i}{j-i}
$$

In our example:

$$
\mathbf{m}_{(5,2)}=\frac{1}{1 \cdot 1 \cdot 2 \cdot 2 \cdot 3 \cdot 5 \cdot 6}=\frac{1 ! \cdot 0 !}{6 ! \cdot 2 !} \cdot \frac{4}{1}=\frac{4}{1} \frac{7}{2} \frac{8}{3} \frac{9}{4} \frac{10}{5} \frac{11}{6} \frac{12}{7} \ldots \frac{3}{1} \frac{4}{2} \frac{5}{3} \ldots
$$




\section{Chern characters.}

The Chern character of the partition $\lambda$ is a generating function for the symmetric functions of the eigen-values $\lambda_{i}-i$ :

$$
\begin{aligned}
\operatorname{ch}_{\lambda}(M, u)= & \sum_{i=1}^{\infty} e^{u\left(M+\frac{1}{2}-i+\lambda_{i}\right)}=\frac{e^{M u}}{e^{\frac{u}{2}}-e^{-\frac{u}{2}}}+\sum_{i=1}^{\infty} e^{u\left(M+\frac{1}{2}-i\right)}\left(e^{u \lambda_{i}}-1\right)= \\
=\frac{1}{u}+M+ & u\left(\frac{M^{2}}{2}-\frac{1}{24}+|\lambda|\right)+ \\
& \quad+\frac{u^{2}}{2}\left(\frac{M^{3}}{3}-\frac{M}{12}+2 M|\lambda|+\sum_{i} \lambda_{i}\left(\lambda_{i}-2 i+1\right)\right)+\ldots
\end{aligned}
$$

the second equality being valid when $\Re \mathrm{e} u>0$. In the gauge theory the main object is the Chern character of the "universal sheaf" $\mathcal{E}$, which is related to (A.3) in the following simple way:

$$
\begin{gathered}
\operatorname{ch}(\mathcal{E})=\left(e^{\frac{\hbar u}{2}}-e^{-\frac{\hbar u}{2}}\right) \operatorname{ch}_{\lambda}\left(\frac{a}{\hbar}, \hbar u\right)= \\
=e^{u a}\left(1+\left(1-e^{-u \hbar}\right) \sum_{i=1}^{\infty} e^{u \hbar(1-i)}\left(e^{u \hbar \lambda_{i}}-1\right)\right)= \\
=\sum_{k=0}^{\infty} \frac{u^{k}}{k !} \operatorname{ch}_{k}(a, \lambda)
\end{gathered}
$$

It is the components $\operatorname{ch}_{k}(a, \lambda)$ of Chern character (A.4) that enter the formula for the statistical weight of the partition $\lambda$ in the ensemble (1.8). For example:

$$
\begin{aligned}
& \operatorname{ch}_{0}(a, \lambda)=1 \\
& \operatorname{ch}_{1}(a, \lambda)=a \\
& \operatorname{ch}_{2}(a, \lambda)=a^{2}+2 \hbar^{2}|\lambda| \\
& \operatorname{ch}_{3}(a, \lambda)=a^{3}+6 \hbar^{2} a|\lambda|+3 \hbar^{3} \sum_{i} \lambda_{i}\left(\lambda_{i}+1-2 i\right)
\end{aligned}
$$

One can also write for the Chern characters

$$
\begin{gathered}
\operatorname{ch}_{k}(a, \lambda)=a^{k}+\sum_{i=1}^{\infty}\left(\left(a+\hbar\left(\lambda_{i}-i+1\right)\right)^{k}-\left(a+\hbar\left(\lambda_{i}-i\right)\right)^{k}-\right. \\
\left.-(a+\hbar(1-i))^{k}+(a-\hbar i)^{k}\right)= \\
=\sum_{i=1}^{\infty}\left(\left(a+\hbar\left(\lambda_{i}-i+1\right)\right)^{k}-\left(a+\hbar\left(\lambda_{i}-i\right)\right)^{k}\right)
\end{gathered}
$$

which is useful to make link with the fermionic formalism. 


\section{References}

[1] A. Losev, A. Marshakov, N. Nekrasov, "Small instantons, little strings, and free fermions", hep-th/0302191, Ian Kogan memorial volume, M.Shifman, A.Vainshtein and J. Wheater (eds.) "From fields to strings: circumnavigating theoretical physics", $581-621$

[2] N. Nekrasov, "Seiberg-Witten prepotential from instanton counting", hep-th/0206161, hep-th/0306211, Adv. Theor. Math. Phys.7 (2004) 831-864

[3] N. Nekrasov, A. Okounkov, "Seiberg-Witten theory and random partitions", hepth/0306238

[4] A. Gorsky, I. Krichever, A. Marshakov, A. Mironov, A. Morozov, "Integrability and Seiberg-Witten exact solution", hep-th/9505035, Phys. Lett. 355B (1995) 466

[5] A. Marshakov, "Seiberg-Witten theory and integrable systems," World Scientific, Singapore (1999);

"Integrability: the Seiberg-Witten and Whitham equations", Eds. H. Braden and I. Krichever, Gordon and Breach (2000)

[6] I. Krichever, "The $\tau$-function of the universal Whitham hierarchy, matrix models and topological field theories", hep-th/9205110, Commun. Pure. Appl. Math. 47 (1992) 437.

[7] A. Losev, N. Nekrasov, S. Shatashvili, "Issues in topological gauge theory", hepth/9711108, Nucl. Phys. B534 (1998) 549-611

[8] A. Gorsky, A. Marshakov, A. Mironov, A. Morozov, "RG equations from Whitham hierarchy", hep-th/9802007, Nucl. Phys. B527 (1998) 690-716

[9] N. Nekrasov, A. Schwarz, hep-th/9802068

[10] A. Losev, N. Nekrasov, S. Shatashvili, "The freckled instantons", hep-th/9908204, Yuri Golfand Memorial Volume, Shifman M.A. (ed.) "The many faces of the superworld", 453-475, World Scientific; "Freckled instantons in two and four dimensions", hepth/9911099, Class. Quant. Grav. 17(2000) 1181-1187

[11] T. Hollowood, "Calculating the prepotential by localization on the moduli space of instantons", hep-th/0201075; "Testing Seiberg-Witten theory to all orders in the instanton expansion", hep-th/0202197

[12] K. Intriligator, P. Kraus, A. Ryzhov, M. Shigemori, C. Vafa, "On low rank classical groups in string theory, gauge theory and matrix models", hep-th/0311181, Nucl. Phys. B682 (2004) 45-82

[13] B.F. Logan, L.A. Shepp, "A variational problem for random Young tableaux", Advances in Math. 26 (1977), no. 2, 206-222

[14] S. V. Kerov, A. M. Vershik, "Asymptotics of the Plancherel measure of the symmetric group and the limit shape of the Young diagrams", ДАН СССР, 233 (1977), no. 6, 1024-1027 (in Russian) 
[15] S. V. Kerov, A. M. Vershik, "Asymptotic behaviour of the maximum and generic dimensions of irreducible representations of the symmetric group," (Russian) Функ. Анал. и Приложен. 19 (1985), nо. 1, 25-36

[16] S.V. Kerov, "Gaussian limit for the Plancherel measure of the symmetric group", C. R. Acad. Sci. Paris Sr. I Math. 316 (1993), no. 4, 303-308.

[17] T. Eguchi, S.-K. Yang, "The topological $\mathbf{C P}^{1}$ model and the large- $N$ matrix integral", Mod. Phys. Lett. A9 (1994) 2893-2902, hep-th/9407134

[18] G. Moore, N. Nekrasov, S. Shatashvili, "Integration over the Higgs branches", hepth/9712241, Comm. Math. Phys. 209 (2000) 97-121

[19] A. Marshakov, A. Mironov, A. Morozov, "Generalized matrix models as conformal field theories: Discrete case", Phys. Lett. B265, 99 (1991);

S. Kharchev, A. Marshakov, A. Mironov, A. Morozov, S. Pakuliak, "Conformal matrix models as an alternative to conventional multi-matrix models", hep-th/9208044, Nucl.Phys. B404 (1993) 717-750.

[20] G.Akemann, "Higher genus correlators for the Hermitian matrix model with multiple cuts", hep-th/9606004, Nucl.Phys. B482 (1996) 403;

I. K. Kostov, "Conformal field theory techniques in random matrix models", hepth/9907060;

R.Dijkgraaf, A.Sinkovics, M.Temürhan, "Matrix models and gravitational corrections", hep-th/0211241.

[21] W. -P. Li, Z .Qin, W. Wang, "Hilbert schemes, integrable hierarchies, and GromovWitten theory", Internat. Math. Res. Notices 40 (2004), 2085-2104, math.AG/0302211

[22] K. Takasaki, T. Takebe, "Quasi-classical limit of Toda hierarchy and W-infinity symmetries", hep-th/9301070, Lett. Math. Phys. 28 (1993) 165-176; "Integrable hierarchies and dispersionless limit", hep-th/9405096, Rev.Math.Phys. 7 (1995) 743-808.

[23] T. Maeda, T. Nakatsu, K. Takasaki, T. Tamakoshi, "Free fermion and Seiberg-Witten differential in random plane partitions", hep-th/0412329; "Five-dimensional supersymmetric Yang-Mills theories and random plane partitions", hep-th/0412327

[24] I. Macdonald, "Symmetric functions and Hall polynomials", Clarendon Press, Oxford, 1979

[25] A. Okounkov, R. Pandharipande, The equivariant Gromov-Witten theory of $\mathbf{P}^{1}$, math.AG/0207233; Gromov-Witten theory, Hurwitz theory, and completed cycles, math.AG/0204305

[26] V.A. Novikov, M.A. Shifman, A.I. Vainshtein, V.I. Zakharov, "Beta function in supersymmetric gauge theories: instantons versus traditional approach", Phys. Lett. 166B (1986) 329-333, Sov.J.Nucl.Phys. 43 (1986) 294, Яд.Физ. 43 (1986) 459-464

[27] S. Kharchev and A. Marshakov, "Topological versus non-topological theories and $p-q$ duality in $c \leq 12$ gravity models, hep-th/9210072, in "String theory, quantum gravity 
and the unification of the fundamental interactions", M.Bianchi, F.Fucito, E.Marinari, A.Sagnotti (eds.), World Scientific, 1993, 331-346; Integral representations and $p-q$ duality in $c \leq 1$ 2d gravity, hep-th/9303100, Int. J. Mod. Phys. A10 (1995) 1219.

[28] N. Nekrasov, Lectures on super-Yang-Mills, partitions, Mayer expansion, and limit shapes, UvA, May 2005

[29] A.Marshakov, "Strings, integrable systems, geometry and statistical models", hepth/0401199.

[30] P. B. Wiegmann, A. Zabrodin, "Conformal maps and dispersionless integrable hierarchies," hep-th/9909147, Commun. Math. Phys. 213 (2000) 523;

I. K. Kostov, I. Krichever, M. Mineev-Weinstein, P. B. Wiegmann, A. Zabrodin, “ $\tau$ function for analytic curves," hep-th/0005259

[31] A. Marshakov, P. Wiegmann, A. Zabrodin, "Integrable structure of the Dirichlet boundary problem in two dimensions," hep-th/0109048, Commun. Math. Phys. 227 (2002) 131;

I. Krichever, A. Marshakov, A. Zabrodin, "Integrable structure of the Dirichlet boundary problem in multiply-connected domains," hep-th/0309010, Commun. Math. Phys. 259 (2005) 1

[32] S.V. Kerov, "Interlacing measures", Kirillov's seminar on representation theory, 3583, Amer. Math. Soc. Transl. Ser. 2, 181, Amer. Math. Soc., Providence, RI, 1998; "Anisotropic Young diagrams and symmetric Jack functions", (Russian) Функ. Анал. и Приложен. 34 (2000), no. 1, 51-64, 96; translation in Funct. Anal. Appl. 34 (2000), no. 1, 41-51; "Random Young tableaux", Теор. вероят. и ее применения, 3 (1986), 627-628 (in Russian);

A. M. Vershik, "Hook formulae and related identities", Записки сем. ЛОМИ, 172 (1989), 3-20 (in Russian)

[33] M. Douglas, V. Kazakov, hep-th/9305047;

D. Gross, A. Matytsin, hep-th/9404004

[34] V.I. Arnol'd, "Mathematical methods of classical mechanics", Moscow, Nauka, 1989

[35] J. Fay, "Theta-functions on Riemann surfaces", Lect.Notes Math., Vol. 352, Springer, New York, 1973

[36] S. Kharchev, A. Marshakov, A. Mironov and A. Morozov, "Landau-Ginzburg topological theories in the framework of GKM and equivalent hierarchies", hep-th/9208046, Mod. Phys. Lett. A8, 1047 (1993)

[37] E. Witten, "Mirror manifolds and topological field theory", hep-th/9112056;

M. Kontsevich, "Homological algebra of mirror symmetry", alg-geom/9411018

[38] T. Eguchi, K. Hori, S.-K. Yang, "Topological $\sigma$-models and large-N matrix integral", Int. J. Mod. Phys. A10 (1995) 4203, hep-th/9503017

[39] S. A. Yost, "Supermatrix models", hep-th/9111033, Int. J. Mod. Phys. A7 (1992) 6105 
[40] R. Dijkgraaf, S. Gukov, V. Kazakov and C. Vafa, "Perturbative analysis of gauged matrix models", hep-th/0210238, Phys. Rev. D68 (2003) 045007

[41] V. Kazakov, A. Marshakov, "Complex curve of the two matrix model and its taufunction", hep-th/0211236, J. Phys. A36 (2003) 3107

[42] R. Donagi, E. Witten, "Supersymmetric Yang-Mills theory and integrable systems.", hep-th/9510101, Nucl. Phys. B460 (1996) 299-334

[43] T. Nakatsu, K. Takasaki, "Whitham-Toda hierarchy and $\mathcal{N}=2$ supersymmetric YangMills theory", hep-th/9509162, Mod. Phys. Lett. 11A (1996) 157;

M. Mariño, G. Moore, "The Donaldson-Witten function for gauge groups of rank larger than one", hep-th/9802185, Comm. Math. Phys. 199 (1998) 25-69;

J. Edelstein, M. Mariño, J. Mas, "Whitham hierarchies, instanton corrections and soft supersymmetry breaking in $N=2 S U(N)$ super Yang-Mills theory", hep-th/9805172, Nucl.Phys. B541 (1999) 671

[44] H. Nakajima, K. Yoshioka, "Lectures on instanton counting", math.AG/0311058

[45] A. Okounkov, N. Reshetikhin, math.CO/0107056;

A. Okounkov, R. Kenyon, S. Sheffield, math-ph/0311005;

A. Okounkov, R. Kenyon, math.AG/0311062

[46] E. Bettelheim, A. Abanov, P. Wiegmann, nlin.SI/0605006 